\title{
A Practical Auxiliary for Quaternary Carbon Formation With Chiral Bicyclic Thioglycolate Lactams.
}

Azélie Arpin, Jeffrey M. Manthorpe and James L. Gleason*, Department of Chemistry, McGill University, 801 Sherbrooke Street West, Montréal, QC, Canada, H3A 2K6.

\section{Experimental Section}

General Experimental. All reagents were commercial materials and were used without further purification with the following exceptions. Tetrahydrofuran and diethyl ether were distilled from sodium benzophenone ketyl. Toluene and methylene chloride were distilled from calcium hydride. Valinol can be purchased from Aldrich or synthesized via the methodology published by A. I. Meyers. ${ }^{1}$ Alkylation and reductive enolization substrates were dried via azeotropic distillation of water using dry toluene. All Schlenk flasks and lithium chloride were flame-dried under vacuum. Alkyl halides were passed through basic alumina prior to addition. Chromatography was conducted using 230-400 mesh silica gel. NMR spectra were recorded at 270, 300, 400 or $500 \mathrm{MHz}$ for ${ }^{1} \mathrm{H}$ and $67.5,75$ or $100 \mathrm{MHz}$ for ${ }^{13} \mathrm{C}$. GC analyses were conducted on a CP Chiralsil-dex column $(25 \mathrm{~m} \times 0.25 \mathrm{~mm})$ using $\mathrm{He}(14 \mathrm{psi})$ as carrier gas unless mentionned. Elemental analyses were obtained from Quantitative Technologies Inc., Whitehouse, NJ. High resolution mass spectroscopies were conducted by M. Gaston Boulay at Universite de Sherbrooke.

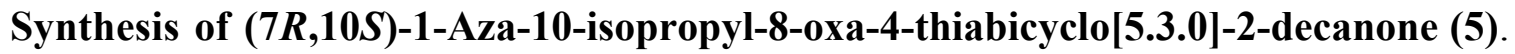

Methyl thioglycolate 4 (12.61 mL, $141.0 \mathrm{mmol}, 2.0$ equiv.) was added slowly to a mixture of 
$60 \% \mathrm{NaH}\left(5.64 \mathrm{~g}, 141.0 \mathrm{mmol}, 2.0\right.$ equiv.) in tetrahydrofuran $(200 \mathrm{~mL})$ at $23{ }^{\circ} \mathrm{C}$ resulting in a vigorous evolution of hydrogen. Once the gas evolution had ceased, 2-(2-bromoethyl)-1,3dioxolane $(8.28 \mathrm{~mL}, 70.5 \mathrm{mmol}, 1.0$ equiv.) was added slowly via a glass syringe. The resulting mixture was stirred for $16 \mathrm{~h}$. Saturated aqueous sodium bicarbonate $(150 \mathrm{~mL})$ was added and the mixture was extracted with ethyl acetate $(3 \mathrm{X} 150 \mathrm{~mL})$. The combined organic layers were dried over anhydrous sodium sulfate, filtered and concentrated. The residue was purified by chromatography on silica gel eluting with $15 \%$ ethyl acetate in hexanes to afford (2[1,3]dioxolan-2-yl-ethylsulfanyl)-acetic acid methyl ester (13.81 g, $67.2 \mathrm{mmol})$ in 95\% yield as a yellow oil. ${ }^{1} \mathrm{H}$ NMR $\left(\mathrm{CDCl}_{3}\right) \delta, 5.00(\mathrm{t}, 1 \mathrm{H}, J=4.5 \mathrm{~Hz}), 3.97(\mathrm{~m}, 2 \mathrm{H}), 3.89(\mathrm{~m}, 2 \mathrm{H}), 3.73(\mathrm{~s}$, $3 \mathrm{H}), 3.24(\mathrm{~s}, 2 \mathrm{H}), 2.74,(\mathrm{t}, 2 \mathrm{H}, J=7.8 \mathrm{~Hz}), 1.98(\mathrm{~m}, 2 \mathrm{H}) ;{ }^{13} \mathrm{C} \mathrm{NMR}\left(\mathrm{CDCl}_{3}\right) \delta, 170.9,103.2$, 65.3, 52.7, 34.0, 33.8, 30.0, 27.5. HRMS calc. for $\mathrm{C}_{8} \mathrm{H}_{14} \mathrm{O}_{4} \mathrm{~S}: 206.0613$. Found: 206.0619 \pm 0.0006 .

A $2.50 \mathrm{M}$ solution $n$-butyllithium in hexanes $(5.38 \mathrm{~mL}, 13.4 \mathrm{mmol}, 0.2$ equiv.) was added to a solution of valinol $(8.31 \mathrm{~g}, 80.7 \mathrm{mmol}, 1.2$ equiv. $)$ in tetrahydrofuran $(150 \mathrm{~mL})$ at $0{ }^{\circ} \mathrm{C}$. The mixture was stirred for 5 minutes, at which time, a solution of (2-[1,3]dioxolan-2-ylethylsulfanyl)-acetic acid methyl ester (13.81 g, $67.2 \mathrm{mmol}, 1.0$ equiv.) in tetrahydrofuran (25 $\mathrm{mL}$ ) was added via cannula. The resulting mixture was stirred for $16 \mathrm{~h}$ at $23{ }^{\circ} \mathrm{C}$. Saturated aqueous ammonium chloride $(150 \mathrm{~mL})$ was added and the mixture was extracted with ethyl acetate $(3 \times 75 \mathrm{~mL})$. The combined organic layers were dried over anhydrous sodium sulfate, filtered and concentrated. The residue was purified by chromatography on silica gel eluting with $60 \%$ ethyl acetate and 5\% methanol in hexanes to afford 2-(2-[1,3]dioxolan-2-yl-ethylsulfanyl)$\mathrm{N}$-(1-hydroxymethyl-2-methyl-propyl)-acetamide $(17.09 \mathrm{~g}, 61.8 \mathrm{mmol})$ in $92 \%$ yield as a yellow oil. ${ }^{1} \mathrm{H}$ NMR $\left(\mathrm{CDCl}_{3}\right)$ d, $7.12(\mathrm{~d}, 1 \mathrm{H}, J=9.2 \mathrm{~Hz}), 4.95(\mathrm{t}, 1 \mathrm{H}, J=3.9 \mathrm{~Hz}), 3.95(\mathrm{~m}, 2 \mathrm{H}), 3.85(\mathrm{~m}$, 
2H), $3.69(\mathrm{~m}, 3 \mathrm{H}), 3.28(\mathrm{~s}, 2 \mathrm{H}), 2.67,(\mathrm{~m}, 2 \mathrm{H}), 2.45(\mathrm{bs}, 1 \mathrm{H}), 1.95(\mathrm{~m}, 3 \mathrm{H}), 0.97(\mathrm{~d}, 3 \mathrm{H}, J=7.0$ $\mathrm{Hz}), 0.95(\mathrm{~d}, 3 \mathrm{H}, J=6.7 \mathrm{~Hz}) ;{ }^{13} \mathrm{C} \mathrm{NMR}\left(\mathrm{CDCl}_{3}\right) \delta, 169.5,103.0,65.3,64.0,57.7,36.9,33.7$, 29.3, 28.5, 19.9, 19.1. HRMS calc. for $\mathrm{C}_{12} \mathrm{H}_{23} \mathrm{NO}_{4} \mathrm{~S}: 277.1348$. Found: $277.1353 \pm 0.0008$.

Boron trifluoride diethyletherate $(9.40 \mathrm{~mL}, 74.2 \mathrm{mmol}, 1.2$ equiv.) was added dropwise to a solution of 2-(2-[1,3]dioxolan-2-yl-ethylsulfanyl)-N-(1-hydroxymethyl-2-methyl-propyl)acetamide $\left(17.09 \mathrm{~g}, 61.8 \mathrm{mmol}, 1.0\right.$ equiv.) in dichloromethane $(150 \mathrm{~mL})$ at $23{ }^{\circ} \mathrm{C}$. After $18 \mathrm{~h}$, saturated aqueous sodium bicarbonate $(75 \mathrm{~mL})$ was added and the layers were separated. The aqueous phase was extracted with dichloromethane $(3 \times 100 \mathrm{~mL})$. The combined organic phases were dried over anhydrous sodium sulfate, filtered and concentrated in vacuo. The product was purified by chromatography on silica gel eluting with $40 \%$ ethyl acetate in hexanes to afford 5 $(11.31 \mathrm{~g}, 52.5 \mathrm{~mol})$ in $85 \%$ yield as a white solid. The product was determined to have $>99 \%$ de by GC chiral analysis: ${ }^{1} \mathrm{H}$ NMR $\left(\mathrm{CDCl}_{3}\right) \delta, 5.26(\mathrm{~d}, 1 \mathrm{H}, J=9.0 \mathrm{~Hz}), 4.18(\mathrm{~m}, 1 \mathrm{H}), 3.95(\mathrm{~m}, 2 \mathrm{H})$, $3.28(\mathrm{~d}, 1 \mathrm{H}, J=14.5 \mathrm{~Hz}), 3.20(\mathrm{dd}, 1 \mathrm{H}, J=1.6,14.5 \mathrm{~Hz}), 2.99(\mathrm{~m}, 1 \mathrm{H}), 2.82$, (m, 1H), $2.48(\mathrm{~m}$, 1H), $2.26(\mathrm{~m}, 1 \mathrm{H}), 2.09(\mathrm{~m}, 1 \mathrm{H}), 0.90(\mathrm{~d}, 3 \mathrm{H}, J=7.0 \mathrm{~Hz}), 0.84(\mathrm{~d}, 3 \mathrm{H}, J=6.7 \mathrm{~Hz}) ;{ }^{13} \mathrm{C}$ NMR $\left(\mathrm{CDCl}_{3}\right) \delta, 168.1,90.54,65.7,62.1,37.0,36.6,30.0,27.9,19.7,16.3 .[\alpha]^{25}=+17.2^{\circ}(\mathrm{c}=6.79$, $\mathrm{CH}_{2} \mathrm{Cl}_{2}$ ). Anal. Calcd for $\mathrm{C}_{10} \mathrm{H}_{17} \mathrm{NO}_{2} \mathrm{~S}: \mathrm{C}, 55.78 ; \mathrm{H}, 7.96 ; \mathrm{N}, 6.51$. Found: $\mathrm{C}, 55.87 ; \mathrm{H}, 7.96 ; \mathrm{N}$, 6.64. $\mathrm{mp}: 138-139^{\circ} \mathrm{C}$.

Rapid synthesis of $(7 R, 10 S)$-1-Aza-10-isopropyl-8-oxa-4-thiabicyclo[5.3.0]-2decanone (5) without purification. Methyl thioglycolate (16.85 mL, $188.0 \mathrm{mmol}, 2.0$ equiv.) was added slowly to a mixture of $60 \% \mathrm{NaH}(7.52 \mathrm{~g}, 188.0 \mathrm{mmol}, 2.0$ equiv.) in tetrahydrofuran $(300 \mathrm{~mL})$ at $23{ }^{\circ} \mathrm{C}$ resulting in a vigorous evolution of hydrogen. Once the gas evolution had ceased, 2-(2-bromoethyl)-1,3-dioxolane (11.04 mL, $94.0 \mathrm{mmol}, 1.0$ equiv.) was added slowly via a glass syringe. The resulting mixture was stirred for $16 \mathrm{~h}$. Saturated aqueous sodium 
bicarbonate $(200 \mathrm{~mL})$ was added and the mixture was extracted with ethyl acetate $(3 \mathrm{X} 150 \mathrm{~mL})$. The combined organic layers were dried over anhydrous sodium sulfate, filtered and concentrated. A $2.50 \mathrm{M}$ solution $n$-butyllithium in hexanes $(7.24 \mathrm{~mL}, 18.1 \mathrm{mmol}, 0.2$ equiv.) was added to a solution of valinol $(11.20 \mathrm{~g}, 108.6 \mathrm{mmol}, 1.2$ equiv. $)$ in tetrahydrofuran $(150 \mathrm{~mL})$ at 0 ${ }^{\circ} \mathrm{C}$. The mixture was stirred for 5 minutes, at which time, a crude solution of $(2-[1,3]$ dioxolan-2yl-ethylsulfanyl)-acetic acid methyl ester (18.57 g, 90.5 mmol, 1.0 equiv.) in tetrahydrofuran (25 $\mathrm{mL}$ ) was added via cannula. The resulting mixture was stirred for $16 \mathrm{~h}$ at $23{ }^{\circ} \mathrm{C}$. Saturated aqueous ammonium chloride $(150 \mathrm{~mL})$ was added and the mixture was extracted with ethyl acetate $(3 \times 75 \mathrm{~mL})$. The combined organic layers were dried over anhydrous sodium sulfate, filtered and concentrated. Boron trifluoride diethyletherate (13.08 mL, $103.2 \mathrm{mmol}, 1.2$ equiv.) was added dropwise to a crude solution of 2-(2-[1,3]dioxolan-2-yl-ethylsulfanyl)-N-(1hydroxymethyl-2-methyl-propyl)-acetamide ( $23.77 \mathrm{~g}, 86.0 \mathrm{mmol}, 1.0$ equiv.) in dichloromethane $(150 \mathrm{~mL})$ at $23{ }^{\circ} \mathrm{C}$. After $18 \mathrm{~h}$, saturated aqueous sodium bicarbonate $(100 \mathrm{~mL})$ was added and the layers were separated. The aqueous phase was extracted with dichloromethane ( 3 x $100 \mathrm{~mL})$. The combined organic phases were dried over anhydrous sodium sulfate, filtered and concentrated in vacuo. The product was crystallized out from diethyl ether to afford 5 (13.36 g, $62.0 \mathrm{mmol}$ ) in $71 \%$ yield as a white solid. The product was determined to have $>99 \%$ de by GC chiral analysis.

Sample procedure for alkylation with alkyl halides: Synthesis of $(3 S, 7 R, 10 S)-1-A z a-$ 3-ethyl-10-isopropyl-8-oxa-4-thiabicyclo[5.3.0]-2-decanone (6a): A solution of $n$-butyllithium in hexanes (2.50 M, $204 \mu \mathrm{L}, 1.10$ equiv.) was added to a slurry of diisopropylamine ( $74.8 \mu \mathrm{L}$, $.53 \mathrm{mmol}, 1.15$ equiv.) and lithium chloride ( $98.0 \mathrm{mg}, 2.32 \mathrm{mmol}, 5$ equiv.) in tetrahydrofuran (4 $\mathrm{mL})$ at $0{ }^{\circ} \mathrm{C}$. After 5 minutes, a solution of $(7 S, 10 S)$-1-aza-10-isopropyl-8-oxa-4- 
thiabicyclo[5.3.0]-2-decanone 5 (100 $\mathrm{mg}, 0.46 \mathrm{mmol}, 1.0$ equiv.) in tetrahydrofuran $(6 \mathrm{~mL})$ was added via cannula. The resulting mixture was stirred for 15 minutes, at which time, ethyl iodide (74.5 $\mu \mathrm{L}, .93 \mathrm{mmol}, 2.0$ equiv.) was added dropwise. Stirring was continued for $12 \mathrm{~h}$ at $0{ }^{\circ} \mathrm{C}$. Saturated aqueous ammonium chloride solution $(10 \mathrm{~mL})$ was added and the resulting mixture was warmed to $23{ }^{\circ} \mathrm{C}$ and extracted with ethyl acetate $(3 \mathrm{X} 15 \mathrm{~mL})$. The combined organic layers were dried over anhydrous sodium sulfate, filtered and concentrated. The residue was purified by chromatography on silica gel eluting with $40 \%$ ethyl acetate in hexanes to afford $(3 S, 7 S, 10 S)$-1-aza-3-ethyl-10-isopropyl-[8-oxa-4-thiabicyclo[5.3.0]-2-decanone 6a as a white solid (106 mg, .44 mmol) in 95\% yield. The product was determined to have $>99 \%$ de by GC chiral analysis: ${ }^{1} \mathrm{H}$ NMR $\left(\mathrm{CDCl}_{3}\right) \delta, 5.39(\mathrm{dd}, 1 \mathrm{H}, J=1.6,10.2 \mathrm{~Hz}), 4.28(\mathrm{~m}, 1 \mathrm{H}), 3.89(\mathrm{~m}, 2 \mathrm{H})$, $3.34(\mathrm{t}, 1 \mathrm{H}, J=7.5 \mathrm{~Hz}), 2.97-2.63(\mathrm{~m}, 2 \mathrm{H}), 2.42,(\mathrm{~m}, 1 \mathrm{H}), 2.30(\mathrm{~m}, 1 \mathrm{H}), 2.14-1.98$ (m, 2H), 1.88 $(\mathrm{m}, 1 \mathrm{H}), 1.07,(\mathrm{t}, 3 \mathrm{H}, J=7.2 \mathrm{~Hz}), 0.89(\mathrm{~d}, 3 \mathrm{H}, J=7.0 \mathrm{~Hz}), 0.84(\mathrm{~d}, 3 \mathrm{H}, J=7.0 \mathrm{~Hz}) ;{ }^{13} \mathrm{C} \mathrm{NMR}$ $\left(\mathrm{CDCl}_{3}\right) \delta, 170.0,88.8,64.5,62.9,50.0,35.8,28.1,23.7,23.5,19.6,16.3,12.1$. HRMS calc. for $\mathrm{C}_{12} \mathrm{H}_{21} \mathrm{NO}_{2} \mathrm{~S}: 243.1293$. Found: $243.1297 \pm 0.00087$. mp: $106-107^{\circ} \mathrm{C}$.

\section{Synthesis of $(3 S, 7 R, 10 S)$-1-Aza-10-isopropyl-3-methyl-8-oxa-4-thiabicyclo [5.3.0]-2-}

decanone (6b): A solution of $n$-butyllithium in hexanes (2.50 M, $2.04 \mathrm{~mL}, 1.10$ equiv.) was added to a slurry of diisopropylamine ( $748 \mu \mathrm{L}, 5.3 \mathrm{mmol}, 1.15$ equiv.) and lithium chloride (984 $\mathrm{mg}, 23.2 \mathrm{mmol}, 5$ equiv.) in tetrahydrofuran $(10 \mathrm{~mL})$ at $0{ }^{\circ} \mathrm{C}$. After 5 minutes, a solution of (7S,10S)-1-aza-10-isopropyl-8-oxa-4-thiabicyclo[5.3.0]-2-decanone 5 (1.00 g, $4.6 \mathrm{mmol}, 1.0$ equiv.) in tetrahydrofuran $(20 \mathrm{~mL})$ was added via cannula. The resulting mixture was stirred for 15 minutes, at which time, methyl iodide ( $376 \mu \mathrm{L}, 6.0 \mathrm{mmol}, 1.2$ equiv.) was added dropwise. Stirring was continued for $12 \mathrm{~h}$ at $0{ }^{\circ} \mathrm{C}$. Saturated aqueous ammonium chloride solution $(25 \mathrm{~mL})$ was added and the resulting mixture was warmed to $23{ }^{\circ} \mathrm{C}$ and extracted with ethyl acetate $(3 \mathrm{X}$ 
$25 \mathrm{~mL}$ ). The combined organic layers were dried over anhydrous sodium sulfate, filtered and concentrated. The residue was purified by chromatography on silica gel eluting with $40 \%$ ethyl acetate in hexanes to afford $(3 S, 7 S, 10 S)$-1-aza-10-isopropyl-3-methyl-8-oxa-4thiabicyclo[5.3.0]-2-decanone $6 \mathbf{b}(0.96 \mathrm{~g}, 4.2 \mathrm{mmol})$ in $90 \%$ yield as a white solid. The product was determined to have $>98 \%$ de by GC chiral analysis: ${ }^{1} \mathrm{H}$ NMR $\left(\mathrm{CDCl}_{3}\right) \delta, 5.32(\mathrm{~d}, 1 \mathrm{H}, J=9.8$ Hz), $4.28(\mathrm{~m}, 1 \mathrm{H}), 3.85(\mathrm{~m}, 2 \mathrm{H}), 3.57$ (q, 1H, $J=7.4 \mathrm{~Hz}), 2.90-2.77(\mathrm{~m}, 2 \mathrm{H}), 2.40,(\mathrm{~m}, 2 \mathrm{H}), 2.06$ (m, 1H), 1.59 (d, 3H, $J=7.8 \mathrm{~Hz}), 0.88(\mathrm{~d}, 3 \mathrm{H}, J=6.7 \mathrm{~Hz}), 0.85(\mathrm{~d}, 3 \mathrm{H}, J=7.0 \mathrm{~Hz}) ;{ }^{13} \mathrm{C}$ NMR $\left(\mathrm{CDCl}_{3}\right) \delta, 170.8,88.9,64.7,62.6,41.7,35.0,28.2,24.2,19.3,17.2,16.4$. Anal. Calcd for $\mathrm{C}_{11} \mathrm{H}_{19} \mathrm{NO}_{2} \mathrm{~S}: \mathrm{C}, 57.61 ; \mathrm{H}, 8.35 ; \mathrm{N}, 6.11$. Found: $\mathrm{C}, 57.68 ; \mathrm{H}, 8.42 ; \mathrm{N}, 6.09 . \mathrm{mp}: 107-108{ }^{\circ} \mathrm{C}$.

$(3 S, 7 R, 10 S)$-1-Aza-10-isopropyl-3-propyl-8-oxa-4-thiabicyclo[5.3.0]-2-decanone (6c):

The alkylation was carried out following the general procedure above starting from 5 (100 $\mathrm{mg}$, $.46 \mathrm{mmol}$ ) at $0{ }^{\circ} \mathrm{C}$ over $16 \mathrm{~h}$ using 2.0 equiv. of propyl iodide. The residue was purified by chromatography on silica gel eluting with $40 \%$ ethyl acetate in hexanes to afford $\mathbf{6 c}$ (106 mg, .41 $\mathrm{mmol}$ ) as a white solid in $89 \%$ yield. The product was determined to have $>99 \%$ de by GC chiral analysis: ${ }^{1} \mathrm{H}$ NMR $\left(\mathrm{CDCl}_{3}\right)$ d, $5.41(\mathrm{~d}, 1 \mathrm{H}, J=10.2 \mathrm{~Hz}), 4.27(\mathrm{~m}, 1 \mathrm{H}), 3.87(\mathrm{~m}, 2 \mathrm{H}), 3.42(\mathrm{t}, 1 \mathrm{H}$, $J=7.4 \mathrm{~Hz}), 2.94(\mathrm{~m}, 1 \mathrm{H}), 2.64,(\mathrm{~m}, 1 \mathrm{H}), 2.42(\mathrm{~m}, 1 \mathrm{H}), 2.02(\mathrm{~m} .2 \mathrm{H}), 1.82(\mathrm{~m}, 1 \mathrm{H}), 1.52,(\mathrm{~m}$, 1H), $1.41(\mathrm{~m}, 1 \mathrm{H}), 0.98$ (t, 3H, $J=7.0 \mathrm{~Hz}), 0.89$ (d, 3H, $J=7.0 \mathrm{~Hz}), 0.89$ (d, 3H, $J=7.0 \mathrm{~Hz}$ ); ${ }^{13} \mathrm{C} \mathrm{NMR}\left(\mathrm{CDCl}_{3}\right) \delta, 170.0,88.7,64.5,62.8,48.2,35.9,32.2,28.1,23.7,20.8,19.6,16.4,14.1$. Anal. Calcd for $\mathrm{C}_{13} \mathrm{H}_{23} \mathrm{NO}_{2} \mathrm{~S}$ : C, 60.66; H, 9.01; N, 5.44. Found: C, 60.79; H, 9.12; N, 5.41. mp: $77-78^{\circ} \mathrm{C}$.

(3S,7R,10S)-1-Aza-3-benzyl-10-isopropyl-8-oxa-4-thiabicyclo[5.3.0]-2-decanone (6d):

The alkylation was carried out following the general procedure above starting from 5 (100 $\mathrm{mg}$, $.46 \mathrm{mmol}$ ) at $0{ }^{\circ} \mathrm{C}$ over $16 \mathrm{~h}$ using 1.2 equiv. of benzyl bromide. The residue was purified by 
chromatography on silica gel eluting with $40 \%$ ethyl acetate in hexanes to afford $\mathbf{6 d}$ (125 mg, .41 mmol) as a white solid in $88 \%$ yield. The product was determined to have $97 \%$ de by GC chiral analysis: ${ }^{1} \mathrm{H} \mathrm{NMR}\left(\mathrm{CDCl}_{3}\right)$ $\delta, 7.30-7.18(\mathrm{~m} .5 \mathrm{H}), 5.33(\mathrm{dd}, 1 \mathrm{H}, J=1.8,9.6 \mathrm{~Hz}), 4.19(\mathrm{~m}, 1 \mathrm{H})$, $3.95(\mathrm{~m}, 2 \mathrm{H}), 3.39(\mathrm{~m}, 2 \mathrm{H}), 3.10(\mathrm{~m}, 1 \mathrm{H}), 2.88-2.73(\mathrm{~m}, 2 \mathrm{H}), 2.36(\mathrm{~m}, 1 \mathrm{H}), 2.20-2.01(\mathrm{~m}, 2 \mathrm{H})$, $0.84(\mathrm{~d}, 3 \mathrm{H}, J=7.0 \mathrm{~Hz}), 0.73(\mathrm{~d}, 3 \mathrm{H}, J=6.8 \mathrm{~Hz}) ;{ }^{13} \mathrm{C} \mathrm{NMR}\left(\mathrm{CDCl}_{3}\right) \delta, 169.2,139.2,129.3$, 128.4, 126.7, 90.2, 65.7, 61.8, 49.2, 36.8, 36.2, 30.5, 28.3, 19.6, 16.2. HRMS calc. for $\mathrm{C}_{17} \mathrm{H}_{23} \mathrm{NO}_{2} \mathrm{~S}: 305.1449$. Found: $305.1456 \pm 0.0009 . \mathrm{mp}: 135.5-136^{\circ} \mathrm{C}$.

\section{$(3 R, 7 R, 10 S)$-1-Aza-3-ethyl-10-isopropyl-3-methyl-8-oxa-4-thiabicyclo[5.3.0]-2-}

decanone (7a): The alkylation was carried out following the general procedure above starting from 6a (100 mg, $.41 \mathrm{mmol})$ at $0{ }^{\circ} \mathrm{C}$ over $16 \mathrm{~h}$ using 1.2 equiv. of methyl iodide. The residue was purified by chromatography on silica gel eluting with $40 \%$ ethyl acetate in hexanes to afford 7 a $(95 \mathrm{mg}, .37 \mathrm{mmol})$ as a colourless oil in $90 \%$ yield. The product was determined to have $>99 \%$ de by GC chiral analysis: ${ }^{1} \mathrm{H}$ NMR $\left(\mathrm{CDCl}_{3}\right) \delta, 5.64(\mathrm{dd}, 1 \mathrm{H}, J=2.3,9.8 \mathrm{~Hz}), 4.28(\mathrm{~m}$, 1H), 3.93-3.81 (m, 2H), 2.85-2.67 (m, 2H), 2.42-2.25 (m, 2H), $1.93(\mathrm{~m}, 3 \mathrm{H}), 1.51(\mathrm{~s}, 3 \mathrm{H}), 1.06$ $(\mathrm{t}, 3 \mathrm{H}, J=7.4 \mathrm{~Hz}), 0.88(\mathrm{~d}, 3 \mathrm{H}, J=7.0 \mathrm{~Hz}), 0.84\left(\mathrm{~d}, 3 \mathrm{H}, J=6.7 \mathrm{~Hz} ;{ }^{13} \mathrm{C} \mathrm{NMR}\left(\mathrm{CDCl}_{3}\right) \delta, 172.5\right.$, 88.9, 64.4, 62.9, 52.6, 34.7, 33.2, 27.8, 24.8, 24.6, 19.5, 16.3, 9.4. HRMS calc. for $\mathrm{C}_{13} \mathrm{H}_{23} \mathrm{NO}_{2} \mathrm{~S}$ : 257.1449. Found: $257.1454 \pm 0.0008$.

\section{(3S,7R,10S)-1-Aza-3-ethyl-10-isopropyl-3-propyl-8-oxa-4-thiabicyclo[5.3.0]-2-}

decanone (7b): The alkylation was carried out following the general procedure above starting from 6a (100 mg, $.41 \mathrm{mmol})$ at $0{ }^{\circ} \mathrm{C}$ over $16 \mathrm{~h}$ using 2.0 equiv. of propyl iodide. The residue was purified by chromatography on silica gel eluting with $40 \%$ ethyl acetate in hexanes to afford $7 \mathbf{b}(108 \mathrm{mg}, .38 \mathrm{mmol})$ as a white solid in $92 \%$ yield. The product was determined to have $>99 \%$ de by GC chiral analysis: ${ }^{1} \mathrm{H}$ NMR $\left(\mathrm{CDCl}_{3}\right) \delta, 5.72(\mathrm{dd}, 1 \mathrm{H}, J=2.7,9.8 \mathrm{~Hz}), 4.28(\mathrm{~m}, 1 \mathrm{H}), 3.88$ 
(m, 2H), 2.85-2.64 (m, 2H), $2.43(\mathrm{~m}, 1 \mathrm{H}), 2.22(\mathrm{~m}, 1 \mathrm{H}), 2.00-1.77(\mathrm{~m}, 5 \mathrm{H}), 1.37-1.25(\mathrm{~m}, 2 \mathrm{H})$ $1.03(\mathrm{t}, 3 \mathrm{H}, J=7.4 \mathrm{~Hz}), 0.88(\mathrm{~d}, 3 \mathrm{H}, J=7.0 \mathrm{~Hz}), 0.85(\mathrm{~d}, 3 \mathrm{H}, J=7.0 \mathrm{~Hz}) ;{ }^{13} \mathrm{C} \mathrm{NMR}\left(\mathrm{CDCl}_{3}\right) \delta$, 171.3, 88.7, 63.7, 63.0, 57.2, 39.8, 34.2, 30.9, 27.4, 24.1, 19.7, 18.2, 16.0, 14.8, 9.1. Anal. Calcd for $\mathrm{C}_{15} \mathrm{H}_{27} \mathrm{NO}_{2} \mathrm{~S}: \mathrm{C}, 63.12 ; \mathrm{H}, 9.53 ; \mathrm{N}, 4.91$. Found: $\mathrm{C}, 63.29 ; \mathrm{H}, 9.60 ; \mathrm{N}, 4.90 . \mathrm{mp}: 58-59^{\circ} \mathrm{C}$.

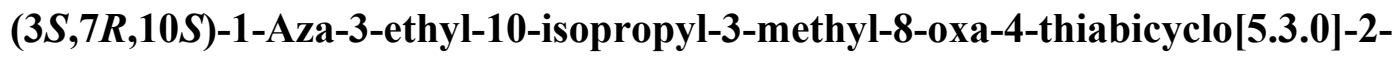

decanone (7c): The alkylation was carried out following the general procedure above starting from $\mathbf{6 b}(100 \mathrm{mg}, .44 \mathrm{mmol})$ at $0{ }^{\circ} \mathrm{C}$ over $16 \mathrm{~h}$ using 2.0 equiv. of ethyl iodide. The residue was purified by chromatography on silica gel eluting with $40 \%$ ethyl acetate in hexanes to afford $7 \mathbf{c}$ ( $97 \mathrm{mg}, .37 \mathrm{mmol}$ ) as a white solid in $86 \%$ yield. The product was determined to have $>99 \%$ de by GC chiral analysis: ${ }^{1} \mathrm{H}$ NMR $\left(\mathrm{CDCl}_{3}\right) \delta, 5.55(\mathrm{dd} .1 \mathrm{H}, J=1.9,9.8 \mathrm{~Hz}), 4.31(\mathrm{~m}, 1 \mathrm{H}), 3.85(\mathrm{~m}$, 2H), $2.93(\mathrm{~m}, 1 \mathrm{H}), 2.69(\mathrm{~m}, 1 \mathrm{H}), 2.40(\mathrm{~m}, 1 \mathrm{H}), 2.25(\mathrm{~m}, 1 \mathrm{H}), 2.05-1.87(\mathrm{~m}, 3 \mathrm{H}), 1.49(\mathrm{~s}, 3 \mathrm{H})$, $0.97(\mathrm{t}, 3 \mathrm{H}, J=7.4 \mathrm{~Hz}), 0.88(\mathrm{~d}, 3 \mathrm{H}, J=7.0 \mathrm{~Hz}), 0.85(\mathrm{~d}, 3 \mathrm{H}, J=7.0 \mathrm{~Hz}) ;{ }^{13} \mathrm{C} \mathrm{NMR}\left(\mathrm{CDCl}_{3}\right) \delta$, 172.0, 89.1, 63.9, 63.5, 51.8, 35.4, 27.8, 25.9, 24.1, 19.8, 16.2, 9.3. Anal. Calcd for $\mathrm{C}_{13} \mathrm{H}_{23} \mathrm{NO}_{2} \mathrm{~S}$ : C, 60.66; H, 9.01; N, 5.44. Found: C, 60.70; H, 8.99; N, 5.43. mp: 106-107.5 ${ }^{\circ} \mathrm{C}$.

\section{$(3 R, 7 R, 10 S)$-1-Aza-3-ethyl-10-isopropyl-3-propyl-8-oxa-4-thiabicyclo[5.3.0]-2-}

decanone (7d): The alkylation was carried out following the general procedure above starting from $6 \mathbf{c}(100 \mathrm{mg}, .39 \mathrm{mmol})$ at $0{ }^{\circ} \mathrm{C}$ over $16 \mathrm{~h}$ using 2.0 equiv. of ethyl iodide. The residue was purified by chromatography on silica gel eluting with $40 \%$ ethyl acetate in hexanes to afford $7 \mathbf{d}$ (99 mg, .35 mmol) as colorless oil in $89 \%$ yield. The product was determined to have $94 \%$ de by GC chiral analysis: ${ }^{1} \mathrm{H}$ NMR $\left(\mathrm{CDCl}_{3}\right) \delta, 5.70(\mathrm{dd}, 1 \mathrm{H}, J=2.7,9.8 \mathrm{~Hz}), 4.28(\mathrm{~m}, 1 \mathrm{H}), 3.86(\mathrm{~m}$, 2H), 2.86-2.64 (m, 2H), $2.44(\mathrm{~m}, 1 \mathrm{H}), 2.23(\mathrm{~m}, 1 \mathrm{H}), 1.99-1.74(\mathrm{~m}, 5 \mathrm{H}), 1.50(\mathrm{~m}, 3 \mathrm{H}), 0.98-0.84$ $(\mathrm{m}, 11 \mathrm{H}) ;{ }^{13} \mathrm{C} \mathrm{NMR}\left(\mathrm{CDCl}_{3}\right) \delta, 171.1,88.7,63.7,63.1,57.1,40.2,34.3,30.9,27.5,24.2,19.7$, 17.9, 16.0, 14.9, 9.4. HRMS calc. for $\mathrm{C}_{15} \mathrm{H}_{27} \mathrm{NO}_{2} \mathrm{~S}: 285.1762$. Found: $285.1769 \pm 0.0008$. 


\section{$(3 S, 7 R, 10 S)$-1-Aza-3-benzyl-10-isopropyl-3-methyl-8-oxa-4-thiabicyclo[5.3.0]-2-}

decanone (7e): The alkylation was carried out following the general procedure above starting from $6 \mathbf{d}(100 \mathrm{mg}, .33 \mathrm{mmol})$ at $0{ }^{\circ} \mathrm{C}$ over $16 \mathrm{~h}$ using 1.2 equiv. of methyl iodide. The residue was purified by chromatography on silica gel eluting with $40 \%$ ethyl acetate in hexanes to afford 7e $(99 \mathrm{mg}, .31 \mathrm{mmol})$ as colorless oil in $95 \%$ yield. The product was determined to have $>99 \%$ de by GC chiral analysis: ${ }^{1} \mathrm{H}$ NMR $\left(\mathrm{CDCl}_{3}\right) \delta, 7.37-7.21(\mathrm{~m}, 5 \mathrm{H}), 5.73(\mathrm{dd}, 1 \mathrm{H}, J=2.5,9.6 \mathrm{~Hz})$, $4.30(\mathrm{~m}, 1 \mathrm{H}), 3.86(\mathrm{~m}, 2 \mathrm{H}), 3.36(\mathrm{~d}, 1 \mathrm{H}, J=13.7 \mathrm{~Hz}), 3.10(\mathrm{~d}, 1 \mathrm{H}, J=13.7 \mathrm{~Hz}), 2.64(\mathrm{~m}, 1 \mathrm{H})$, $2.44(\mathrm{~m}, 1 \mathrm{H}), 2.20(\mathrm{~m}, 1 \mathrm{H}), 1.85(\mathrm{~m}, 1 \mathrm{H}), 1.48(\mathrm{~s}, 3 \mathrm{H}), 0.90(\mathrm{~d}, 3 \mathrm{H}, J=7.0 \mathrm{~Hz}), 0.85(\mathrm{~d}, 3 \mathrm{H}, J=$ $7.0 \mathrm{~Hz}) ;{ }^{13} \mathrm{C} \mathrm{NMR}\left(\mathrm{CDCl}_{3}\right) \delta, 172.0,137.2,131.9,127.9,126.8,88.8,64.2,63.2,54.6,45.6$, 34.2, 27.7, 26.0, 25.0, 19.6, 16.4. HRMS calc. for $\mathrm{C}_{18} \mathrm{H}_{5} \mathrm{NO}_{2} \mathrm{~S}: 319.1606$. Found: 319.1611 \pm 0.0010 .

\section{$(3 R, 7 R, 10 S)-1-A z a-3-b e n z y l-3-e t h y l-10-i s o p r o p y l-8-o x a-4-t h i a b i c y c l o[5.3 .0]-2-$}

decanone (7f): The alkylation was carried out following the general procedure above starting from $6 \mathbf{d}(100 \mathrm{mg}, .33 \mathrm{mmol})$ at $0{ }^{\circ} \mathrm{C}$ over $16 \mathrm{~h}$ using 2.0 equiv. of ethyl iodide. The residue was purified by chromatography on silica gel eluting with $40 \%$ ethyl acetate in hexanes to afford $7 \mathbf{f}$ (101 $\mathrm{mg}, .30 \mathrm{mmol}$ ) as a colorless oil in $92 \%$ yield. The product was determined to have $>99 \%$ de by GC chiral analysis: ${ }^{1} \mathrm{H}$ NMR $\left(\mathrm{CDCl}_{3}\right) \delta, 7.39(\mathrm{~d}, 2 \mathrm{H}, J=8.2 \mathrm{~Hz}), 7.23(\mathrm{~m}, 3 \mathrm{H}), 5.60$ (dd, $1 \mathrm{H}, J=2.7,9.8 \mathrm{~Hz}), 4.29(\mathrm{~m}, 1 \mathrm{H}), 3.86(\mathrm{t}, 1 \mathrm{H}, J=7.0 \mathrm{~Hz}), 3.75(\mathrm{~m}, 1 \mathrm{H}), 3.44(\mathrm{~d}, 1 \mathrm{H}, J=13.6$ Hz), $2.99(\mathrm{~d}, 1 \mathrm{H}, J=13.6 \mathrm{~Hz}), 2.75(\mathrm{~m}, 2 \mathrm{H}), 2.40(\mathrm{~m}, 1 \mathrm{H}), 2.35(\mathrm{~m}, 1 \mathrm{H}), 2.05-4.79(\mathrm{~m}, 3 \mathrm{H})$, $1.06(\mathrm{t}, 3 \mathrm{H}, J=7.4 \mathrm{~Hz}), 0.91(\mathrm{~d}, 3 \mathrm{H}, J=7,0 \mathrm{~Hz}), 0.59(\mathrm{~d}, 3 \mathrm{H}, J=7,0 \mathrm{~Hz}) ;{ }^{13} \mathrm{C} \mathrm{NMR}\left(\mathrm{CDCl}_{3}\right) \delta$, $170.4,137.4,131.9,127.8,126.7,88.5,63.6,63.4,58.7,43.6,33.6,32.1,27.4,24.3,19.9,16.2$, 9.6. HRMS calc. for $\mathrm{C}_{19} \mathrm{H}_{27} \mathrm{NO}_{2} \mathrm{~S}: 333.1762$. Found: $333.1766 \pm 0.0010$. 


\section{Sample procedure for sequential reductive enolization and alkylation of dialkylated}

substrates: [2R,4S]-((2R)-2-Ethyl-2-methyl-2,3-dihydrocinnamoyl)-4-isopropyl-2-(4-phenyl3-thiapropyl)-oxazolidine (9a): Lithium wire (54 mg, $7.8 \mathrm{mmol}, 4.0$ equiv.) was pressed into thin sheets and rinsed in hexanes, tetrahydrofuran, methanol and tetrahydrofuran and then added in liquid ammonia $(25 \mathrm{~mL})$. After 1 hour of stirring at $-78{ }^{\circ} \mathrm{C}$, dialkylated lactam $7 \mathbf{a}(500 \mathrm{mg}$, 1.94 mmol, 1.0 equiv. $)$ in tetrahydrofuran $(8 \mathrm{~mL})$ was cannulated into the lithium-ammonia solution. The resulting mixture was stirred for 10 minutes, at which time benzyl bromide (692 $\mu \mathrm{L}, 5.8 \mathrm{mmol}, 3.0$ equiv.) was added dropwise. Stirring was continued for $4 \mathrm{~h}$ at $-78{ }^{\circ} \mathrm{C}$. Saturated aqueous ammonium chloride solution $(15 \mathrm{~mL})$ was added and the resulting mixture was warmed to $23{ }^{\circ} \mathrm{C}$ and extracted with ethyl acetate $(3 \mathrm{X} 20 \mathrm{~mL})$. The combined organic layers were were dried over anhydrous sodium sulfate, filtered and concentrated. The residue was purified by chromatography on silica gel eluting with 5\% ethyl acetate in hexanes to afford 9a $(613 \mathrm{mg}, 1.78 \mathrm{mmol})$ in $92 \%$ yield as a colorless oil. The product was determined to have 93:7 $\mathrm{dr}$ by analysis of the corresponding carboxylic acid. ${ }^{1} \mathrm{H} \mathrm{NMR}\left(\mathrm{CD}_{3} \mathrm{~S}(\mathrm{O}) \mathrm{CD}_{3}, 100{ }^{\circ} \mathrm{C}\right) \delta$, 7.32-7.18 (m, 10H), $5.45(\mathrm{~d}, 1 \mathrm{H}, J=8.1 \mathrm{~Hz}), 3.98-3.46(\mathrm{~m}, 6 \mathrm{H}), 3.13(\mathrm{~d}, 1 \mathrm{H}, J=13.1 \mathrm{~Hz}), 2.68$ (d, 1H, J=13.1 Hz), $2.45(\mathrm{~m}, 3 \mathrm{H}), 2.06-1.94(\mathrm{~m}, 2 \mathrm{H}), 1.73(\mathrm{~m}, 2 \mathrm{H}), 1.68(\mathrm{~m} .1 \mathrm{H}), 1.19(\mathrm{~s}, 3 \mathrm{H})$, 0.91(t, 3H, $J=7.3 \mathrm{~Hz}), 0.83(\mathrm{~d}, 3 \mathrm{H}, J=6.9 \mathrm{~Hz}), 0.72(\mathrm{~d}, 3 \mathrm{H}, J=6.9 \mathrm{~Hz}) ;{ }^{13} \mathrm{C} \mathrm{NMR}\left(\mathrm{CDCl}_{3}, 23\right.$ $\left.{ }^{\circ} \mathrm{C}\right) \delta, 175.1,138.3,138.2,130.9,130.6,129.1,128.7,127.1,126.5,90.5,66.9,64.7,61.3,49.6$, 47.0, 43.6, 36.7, 33.2, 32.8, 27.3, 22.2, 19.9, 15.9, 9.9. HRMS calc. for $\mathrm{C}_{27} \mathrm{H}_{37} \mathrm{NO}_{2} \mathrm{~S}: 439.2545$. Found: $439.2549 \pm 0.0013$.

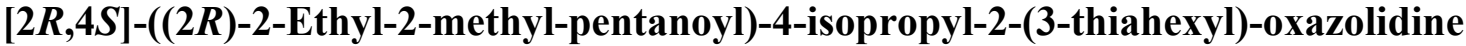

(9b): The reaction was carried out following the general procedure above starting from $7 \mathbf{a}$ (100 $\mathrm{mg}, .39 \mathrm{mmol}$ ) at $-78^{\circ} \mathrm{C}$ over $4 \mathrm{~h}$ using 4.0 equiv. of propyl iodide. The residue was purified by 
chromatography on silica gel eluting with 5\% ethyl acetate in hexanes to afford $9 \mathbf{b}$ (117 $\mathrm{mg}, .34$ mmol) in $88 \%$ yield as a colorless oil. The product was determined to have $99: 1 \mathrm{dr}$ by analysis of the corresponding carboxylic acid. ${ }^{1} \mathrm{H}$ NMR $\left(\mathrm{C}_{6} \mathrm{D}_{6}, 110{ }^{\circ} \mathrm{C}\right) \delta, 5.78(\mathrm{~d}, 1 \mathrm{H}, J=8.1 \mathrm{~Hz}), 3.88$ (m, 1H), $3.51(\mathrm{~m}, 3 \mathrm{H}), 2.56(\mathrm{~m}, 2 \mathrm{H}), 2.35(\mathrm{~m}, 4 \mathrm{H}), 1.75(\mathrm{~m} .3 \mathrm{H}), 1.52(\mathrm{~m}, 2 \mathrm{H}), 1.35(\mathrm{~m}, 3 \mathrm{H})$ $1.19(\mathrm{~s}, 3 \mathrm{H}), 0.88(\mathrm{~m}, 12 \mathrm{H}), 0.60(\mathrm{~d}, 3 \mathrm{H}, J=7.0 \mathrm{~Hz}) ;{ }^{13} \mathrm{C}$ NMR $\left(\mathrm{C}_{6} \mathrm{D}_{6}, 110{ }^{\circ} \mathrm{C}\right) \delta, 175.1,90.4$, $64.3,61.4,48.3,43.3,34.6,34.2,32.5,30.6,27.9,22.9,22.4,19.0,17.7,15.2,14.3,12.8,8.6$. HRMS calc. for $\mathrm{C}_{19} \mathrm{H}_{37} \mathrm{NO}_{2} \mathrm{~S}: 343.2545$. Found: $343.2543 \pm 0.0010$.

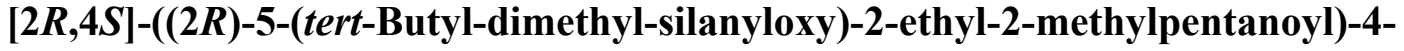
isopropyl-2-(6-(tert-butyl-dimethyl-silanyloxy)-3-thiahexyl)-oxazolidine (9c): The reaction was carried out following the general procedure above starting from $7 \mathbf{a}(100 \mathrm{mg}, .39 \mathrm{mmol})$ at $78{ }^{\circ} \mathrm{C}$ over $4 \mathrm{~h}$ using 4.0 equiv. of tert-butyl-(3-iodo-propoxy)-dimethylsilane. The residue was purified by chromatography on silica gel eluting with $5 \%$ ethyl acetate in hexanes to afford $\mathbf{9 c}$ (200 mg, .34 mmol) in 86\% yield as a yellow oil. The product was determined to have $96: 4 \mathrm{dr}$ by analysis of the corresponding $\delta$-lactone. ${ }^{1} \mathrm{H} \mathrm{NMR}\left(\mathrm{CDCl}_{3}, 23{ }^{\circ} \mathrm{C}\right) \delta, 5.58(\mathrm{~d}, 1 \mathrm{H}, J=9.4 \mathrm{~Hz})$, 4.06-3.90 (m, 3H), 3.70-3.55 (m, 4H), $2.59(\mathrm{~m}, 3 \mathrm{H}), 2.02(\mathrm{~m}, 2 \mathrm{H}), 1.82-1.70(\mathrm{~m}, 4 \mathrm{H}), 1.59-1.42$ $(\mathrm{m}, 4 \mathrm{H}), 1.26(\mathrm{~s}, 3 \mathrm{H}), 0.89(\mathrm{~m}, 27 \mathrm{H}), 0.05(\mathrm{~d}, 12 \mathrm{H}, J=2.3 \mathrm{~Hz}) ;{ }^{13} \mathrm{C} \mathrm{NMR}\left(\mathrm{CDCl}_{3}, 23{ }^{\circ} \mathrm{C}\right) \delta$, $175.6,63.8,61.9,61.3,48.0,37.5,33.1,32.9,29.1,28.4,28.2,26.4,26.3,22.6,20.0,18.7,16.0$, 9.5, -4.9. HRFABMS $m / z 604.4265\left(\mathrm{M}+\mathrm{H}^{+}, \mathrm{C}_{31} \mathrm{H}_{66} \mathrm{NO}_{4} \mathrm{SSi}_{2}{ }^{+}\right.$requires 604.4251).

\section{[2R,4S]-((2R)-6-Chloro-2-ethyl-2-methylhexanoyl)-4-isopropyl-2-(7-chloro-3-}

thiaheptyl)-oxazolidine (9d): The reaction was carried out following the general procedure above starting from $7 \mathbf{a}(100 \mathrm{mg}, .39 \mathrm{mmol})$ at $-78{ }^{\circ} \mathrm{C}$ over $4 \mathrm{~h}$ using 4.0 equiv. of 1-chloro-4iodobutane. The residue was purified by chromatography on silica gel eluting with $5 \%$ ethyl acetate in hexanes to afford $9 \mathbf{d}(136 \mathrm{mg}, .31 \mathrm{mmol})$ in $79 \%$ yield as a yellow oil. The product 
was determined to have 95:5 dr by analysis of the corresponding carboxylic acid. ${ }^{1} \mathrm{H}$ NMR $\left(\mathrm{CDCl}_{3}, 23{ }^{\circ} \mathrm{C}\right) \delta, 5.51(\mathrm{~d}, 1 \mathrm{H}, J=8.6 \mathrm{~Hz}), 4.02-3.81(\mathrm{~m}, 4 \mathrm{H}), 3.45(\mathrm{~m}, 4 \mathrm{H}), 2.47(\mathrm{~m}, 3 \mathrm{H}), 2.04$ $(\mathrm{m}, 2 \mathrm{H}), 1.83-1.14(\mathrm{~m}, 14 \mathrm{H}), 0.83-0.731(\mathrm{~m}, 9 \mathrm{H}) ;{ }^{13} \mathrm{C} \mathrm{NMR}\left(\mathrm{CDCl}_{3}, 23{ }^{\circ} \mathrm{C}\right) \delta, 175.4,90.6,65.2$ $61.3,51.8,48.3,45.3,44.9,33.3,33.2,31.8,31.7,27.9,26.9,22.3,22.2,21.7,20.1,19.9,15.9$, 9.4. HRFABMS $m / z$ 440.2164 $\left(\mathrm{M}+\mathrm{H}^{+}, \mathrm{C}_{21} \mathrm{H}_{40} \mathrm{Cl}_{2} \mathrm{NO}_{2} \mathrm{~S}^{+}\right.$requires 440.2157).

\section{$[2 R, 4 S]-((2 R)-2-E t h y l-2-p r o p y l-p e n t-4-e n o y l)-4-i s o p r o p y l-2-(3-t h i a h e x-5-e n y l)-$}

oxazolidine (9e): The reaction was carried out following the general procedure above starting from $7 \mathbf{d}(100 \mathrm{mg}, .35 \mathrm{mmol})$ at $-78{ }^{\circ} \mathrm{C}$ over $4 \mathrm{~h}$ using 3.0 equiv. of allyl bromide. The residue was purified by chromatography on silica gel eluting with 5\% ethyl acetate in hexanes to afford 9e $(117 \mathrm{mg}, .32 \mathrm{mmol})$ in $91 \%$ yield as a colorless oil. The product was determined to have 95:5 $\mathrm{dr}$ by analysis of the corresponding alcohol. ${ }^{1} \mathrm{H} \mathrm{NMR}\left(\mathrm{CDCl}_{3}, 23{ }^{\circ} \mathrm{C}\right) \delta, 5.76(\mathrm{~m}, 2 \mathrm{H}), 5.48(\mathrm{~d}$, $1 \mathrm{H}, J=8.5 \mathrm{~Hz}), 5.10(\mathrm{~m}, 3 \mathrm{H}), 4.29-3.75(\mathrm{~m}, 7 \mathrm{H}), 3.16(\mathrm{dd}, 2 \mathrm{H}, J=3.5 \mathrm{~Hz}, 7.0 \mathrm{~Hz}), 2.69-1.25$ $(\mathrm{m}, 11 \mathrm{H}), 1.05-0.79(\mathrm{~m}, 12 \mathrm{H}) ;{ }^{13} \mathrm{C} \mathrm{NMR}\left(\mathrm{CDCl}_{3}, 23{ }^{\circ} \mathrm{C}\right) \delta, 174.9,134.4,118.0,117.3,89.5$, $65.3,61.5,51.8,48.3,45.9,39.1,35.2,33.1,28.5,26.8,22.2,20.2,17.9,16.2,15.1,9.3$. HRFABMS $m / z$ 368.2626 $\left(\mathrm{M}+\mathrm{H}^{+}, \mathrm{C}_{21} \mathrm{H}_{38} \mathrm{NO}_{2} \mathrm{~S}^{+}\right.$requires 368.2623).

\section{$[2 R, 4 S]-((2 S)-2-E t h y l-2-m e t h y l-2,3-d i h y d r o c i n n a m o y l)-4-i s o p r o p y l-2-(3-t h i a p e n t y l)-$}

oxazolidine (9f): The reaction was carried out according to the general procedure above starting from $7 \mathrm{e}(100 \mathrm{mg}, .31 \mathrm{mmol})$ at $-78^{\circ} \mathrm{C}$ over $4 \mathrm{~h}$ using 4.0 equiv. of ethyl iodide. The residue was purified by chromatography on silica gel eluting with 5\% ethyl acetate in hexanes to afford 9f (103 $\mathrm{mg}, .27 \mathrm{mmol}$ ) as a colorless oil in $87 \%$ yield. The product was determined to have $97: 3 \mathrm{dr}$ by analysis of the corresponding primary alcohol. ${ }^{1} \mathrm{H} \mathrm{NMR}\left(\mathrm{CDCl}_{3}, 23{ }^{\circ} \mathrm{C}\right) \delta, 7.26-7.14(\mathrm{~m}, 5 \mathrm{H})$, $5.56(\mathrm{~d}, 1 \mathrm{H}, J=9.9 \mathrm{~Hz}), 3.97(\mathrm{~m}, 1 \mathrm{H}), 3.84(\mathrm{~m}, 2 \mathrm{H}), 3.31(\mathrm{~d}, 1 \mathrm{H}, J=12.9 \mathrm{~Hz}), 2.48(\mathrm{~m}, 4 \mathrm{H})$, $2.10(\mathrm{~m}, 1 \mathrm{H}), 1.41(\mathrm{~m}, 1 \mathrm{H}), 1.36(\mathrm{~m} .8 \mathrm{H}), 0.86(\mathrm{t}, 3 \mathrm{H}, J=7.8 \mathrm{~Hz}), 0.70(\mathrm{bs}, 3 \mathrm{H}), 0.61(\mathrm{bs}, 3 \mathrm{H})$ 
${ }^{13} \mathrm{C}$ NMR $\left(\mathrm{CDCl}_{3}, 23{ }^{\circ} \mathrm{C}\right) \delta, 175.0,138.5,130.8,128.3,126.6,90.7,64.8,61.4,50.8,47.9,38.0$, 33.0, 27.6, 26.4, 20.6, 19.8, 16.2, 15.1, 9.4. HRFABMS $m / z$ 378.2473 $\left(\mathrm{M}+\mathrm{H}^{+}, \mathrm{C}_{22} \mathrm{H}_{35} \mathrm{NO}_{2} \mathrm{~S}^{+}\right.$ requires 378.2467).

\section{[2R,4S]-((2S)-2-Ethyl-2-methyl-2,3-dihydrocinnamoyl)-4-isopropyl-2-(4-phenyl-3-}

thiapropyl)-oxazolidine (9g): The reaction was carried out following the general procedure above starting from $7 \mathrm{c}(100 \mathrm{mg}, .39 \mathrm{mmol})$ at $-78{ }^{\circ} \mathrm{C}$ over $4 \mathrm{~h}$ using 3.0 equiv. of benzyl bromide. The residue was purified by chromatography on silica gel eluting with $5 \%$ ethyl acetate in hexanes to afford $9 \mathrm{~g}(162 \mathrm{mg}, .37 \mathrm{mmol})$ as a colorless oil in $85 \%$ yield (the product contained residual dichloromethane). The product was determined to have 93:7 dr by analysis of the corresponding carboxylic acid. ${ }^{1} \mathrm{H}$ NMR $\left(\mathrm{CD}_{3} \mathrm{~S}(\mathrm{O}) \mathrm{CD}_{3}, 100{ }^{\circ} \mathrm{C}\right) \delta, 7.31-7.22(\mathrm{~m}, 10 \mathrm{H}), 5.44$ (d, $1 \mathrm{H}, J=8.1 \mathrm{~Hz}), 3.99-3.70(\mathrm{~m}, 5 \mathrm{H}), 3.17(\mathrm{~d}, 1 \mathrm{H}, J=13.2 \mathrm{~Hz}), 2.45(\mathrm{~m}, 2 \mathrm{H}), 1.91(\mathrm{~m}, 2 \mathrm{H})$, $1.72(\mathrm{~m}, 2 \mathrm{H}), 1.60(\mathrm{~m} .2 \mathrm{H}), 1.21(\mathrm{~s}, 3 \mathrm{H}), 0.83(\mathrm{t}, 3 \mathrm{H}, J=7.3 \mathrm{~Hz}), 0.70(\mathrm{~d}, 3 \mathrm{H}, J=6.8 \mathrm{~Hz}), 0.60$ $(\mathrm{d}, 3 \mathrm{H}, J=6.4 \mathrm{~Hz})) ;{ }^{13} \mathrm{C} \mathrm{NMR}\left(\mathrm{CDCl}_{3}, 23{ }^{\circ} \mathrm{C}\right) \delta, 174.9,138.6,138.5,130.9,130.8,130.6,130.5$, $129.4,129.1,128.7,128.6,128.3,128.2,128.1,127.2,126.6,126.5,66.9,61.4,50.8,47.9,47.0$, $38.0,36.8,33.2,32.6,32.5,27.8,27.5,27.3,20.7,20.5,19.9,15.3,9.9,9.7,9.5$. HRMS calc. for $\mathrm{C}_{27} \mathrm{H}_{37} \mathrm{NO}_{2} \mathrm{~S}: 439.2545$. Found: $439.2549 \pm 0.0013$.

\section{[2R,4S]-((2S)-2-Ethyl-2-methylpentanoyl)-4-isopropyl-2-(3-thiahexyl)-oxazolidine}

(9h): The reaction was carried out following the general procedure above starting from $7 \mathbf{c}$ (100 $\mathrm{mg}, .39 \mathrm{mmol}$ ) at $-78{ }^{\circ} \mathrm{C}$ over $4 \mathrm{~h}$ using 4.0 equiv. of propyl iodide. The residue was purified by chromatography on silica gel eluting with 5\% ethyl acetate in hexanes to afford $\mathbf{9 h}$ (122 $\mathrm{mg}, .36$ mmol) as a colorless oil in 91\% yield. The product was determined to have 99:1 dr by analysis of the corresponding carboxylic acid. ${ }^{1} \mathrm{H}$ NMR $\left(\mathrm{C}_{6} \mathrm{D}_{6}, 90{ }^{\circ} \mathrm{C}\right) \delta, 5.75(\mathrm{dd}, 1 \mathrm{H}, J=1.6,8.6 \mathrm{~Hz})$, $3.91(\mathrm{~m}, 1 \mathrm{H}), 3.67(\mathrm{~m}, 2 \mathrm{H}), 2.57(\mathrm{~m}, 2 \mathrm{H}), 2.41(\mathrm{~m}, 2 \mathrm{H}), 2.24(\mathrm{~m}, 2 \mathrm{H}), 1.77(\mathrm{~m} .3 \mathrm{H}), 1.52(\mathrm{~m}$, 
2H), $1.32(\mathrm{~m}, 3 \mathrm{H}), 1.68(\mathrm{~s}, 3 \mathrm{H}), 0.86(\mathrm{~m}, 12 \mathrm{H}), 0.83(\mathrm{~d}, 3 \mathrm{H}, J=7.0 \mathrm{~Hz}) ;{ }^{13} \mathrm{C} \mathrm{NMR}\left(\mathrm{CDCl}_{3}, 23\right.$

$\left.{ }^{\circ} \mathrm{C}\right) \delta, 175.3,90.1,66.7,64.4,61.3,61.0,48.2,48.1,43.5,42.6,34.5,34.4,34.3,33.1,32.8,30.2$, $30.0,28.0,27.7,23.8,22.9,22.2,22.1,21.1,20.2,19.6,18.7,18.0,17.9,17.8,15.6,14.8,14.7$, 13.5, 10.7, 9.1, 8.0. HRMS calc. for $\mathrm{C}_{19} \mathrm{H}_{37} \mathrm{NO}_{2} \mathrm{~S}: 343.2545$. Found: $343.2543 \pm 0.0010$.

[2R,4S]-((2S)-6-Chloro-2-ethyl-2-methylhexanoyl)-4-isopropyl-2-(7-chloro-3-

thiaheptyl)-oxazolidine (9i): The reaction was carried out following the general procedure above starting from $7 \mathbf{c}(100 \mathrm{mg}, .39 \mathrm{mmol})$ at $-78{ }^{\circ} \mathrm{C}$ over $4 \mathrm{~h}$ using 4.0 equiv. of 1-chloro-4iodobutane. The residue was purified by chromatography on silica gel eluting with $5 \%$ ethyl acetate in hexanes to afford $9 \mathbf{i}(139 \mathrm{mg}, .31 \mathrm{mmol})$ in $81 \%$ yield as a yellow oil. The product was determined to have 95:5 dr by analysis of the corresponding carboxylic acid. ${ }^{1} \mathrm{H}$ NMR $\left(\mathrm{CDCl}_{3}, 23{ }^{\circ} \mathrm{C}\right) \delta, 5.51(\mathrm{~d}, 1 \mathrm{H}, J=8.6 \mathrm{~Hz}), 4.02-3.81(\mathrm{~m}, 4 \mathrm{H}), 3.45(\mathrm{~m}, 4 \mathrm{H}), 2.47(\mathrm{~m}, 3 \mathrm{H}), 2.04$ (m, 2H), 1.83-1.14 (m, 14H), 0.83-0.731 (m, 9H); ${ }^{13} \mathrm{C} \mathrm{NMR}\left(\mathrm{CDCl}_{3}, 23{ }^{\circ} \mathrm{C}\right) \delta, 175.4,90.6,65.2$, $61.3,51.8,48.3,45.3,44.9,33.3,33.2,31.8,31.7,27.9,26.9,22.3,22.2,21.7,20.1,19.9,15.9$, 9.4. HRFABMS $m / z 440.2164\left(\mathrm{M}+\mathrm{H}^{+}, \mathrm{C}_{21} \mathrm{H}_{40} \mathrm{Cl}_{2} \mathrm{NO}_{2} \mathrm{~S}^{+}\right.$requires 440.2157).

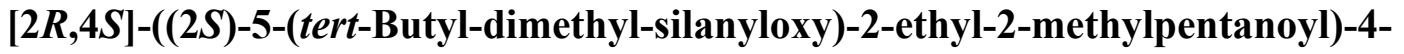
isopropyl-2-(6-(tert-butyl-dimethyl-silanyloxy)-3-thiahexyl)-oxazolidine $(9 \mathrm{j})$ : The reaction was carried out following the general procedure above starting from $7 \mathbf{c}(100 \mathrm{mg}, .39 \mathrm{mmol})$ at $78{ }^{\circ} \mathrm{C}$ over $4 \mathrm{~h}$ using 4.0 equiv. of tert-butyl-(3-iodo-propoxy)-dimethylsilane. The residue was purified by chromatography on silica gel eluting with $5 \%$ ethyl acetate in hexanes to afford $\mathbf{9 j}$ (199 $\mathrm{mg}, .33 \mathrm{mmol}$ ) in $84 \%$ yield as a yellow oil. The product was determined to have 95:5 $\mathrm{dr}$ by analysis of the corresponding $\delta$-lactone. ${ }^{1} \mathrm{H} \mathrm{NMR}\left(\mathrm{CDCl}_{3}, 23{ }^{\circ} \mathrm{C}\right) \delta, 5.56(\mathrm{~d}, 1 \mathrm{H}, J=9.3 \mathrm{~Hz})$, 4.03-3.89 (m, 3H), 3.70-3.57 (m, 4H), $2.58(\mathrm{~m}, 3 \mathrm{H}), 2.01(\mathrm{~m}, 2 \mathrm{H}), 1.82-1.70(\mathrm{~m}, 4 \mathrm{H}), 1.59-1.42$ (m, 4H), $1.26(\mathrm{~s}, 3 \mathrm{H}), 0.89(\mathrm{~m}, 27 \mathrm{H}), 0.05(\mathrm{~d}, 12 \mathrm{H}, J=2.3 \mathrm{~Hz}) ;{ }^{13} \mathrm{C} \mathrm{NMR}\left(\mathrm{CDCl}_{3}, 23{ }^{\circ} \mathrm{C}\right) \delta$, 
$175.5,63.8,61.8,61.3,48.0,37.5,33.1,32.9,29.1,28.4,28.2,26.4,26.3,22.6,20.0,18.7,16.0$,

9.5, -4.8. HRFABMS $m / z 604.4265\left(\mathrm{M}+\mathrm{H}^{+}, \mathrm{C}_{31} \mathrm{H}_{66} \mathrm{NO}_{4} \mathrm{SSi}_{2}{ }^{+}\right.$requires 604.4251).

\section{[2R,4S]-((2S)-2-Ethyl-2-propyl-pent-4-enoyl)-4-isopropyl-2-(3-thiahex-5-enyl)-}

oxazolidine (9k): The reaction was carried out following the general procedure above starting from $7 \mathbf{b}(100 \mathrm{mg}, .35 \mathrm{mmol})$ at $-78{ }^{\circ} \mathrm{C}$ over $4 \mathrm{~h}$ using 3.0 equiv. of allyl bromide. The residue was purified by chromatography on silica gel eluting with $5 \%$ ethyl acetate in hexanes to afford $9 \mathrm{k}(115 \mathrm{mg}, .31 \mathrm{mmol})$ in $89 \%$ yield as a colorless oil. The product was determined to have $96: 4$ dr by analysis of the corresponding alcohol. ${ }^{1} \mathrm{H}$ NMR $\left(\mathrm{CDCl}_{3}, 23{ }^{\circ} \mathrm{C}\right) \delta, 5.78-5.58(\mathrm{~m}, 3 \mathrm{H}), 5.06$ (m, 4H), 4.05-3.85 (m, 4H), $3.14(\mathrm{~d}, 2 \mathrm{H}, J=7.0 \mathrm{~Hz}), 2.59-2.38(\mathrm{~m}, 4 \mathrm{H}), 2.18-2.04(\mathrm{~m}, 3 \mathrm{H})$, 1.71-1.52 (m, 2H), $1.25(\mathrm{~m}, 3 \mathrm{H}), 0.92-0.82(\mathrm{~m}, 9 \mathrm{H}) ;{ }^{13} \mathrm{C} \mathrm{NMR}\left(\mathrm{CDCl}_{3}, 23{ }^{\circ} \mathrm{C}\right) \delta, 174.9,134.5$, 118.1, 117.3, 88.4 65.3, 61.5, 51.8, 48.3, 45.9, 39.1, 38.2, 35.2, 28.5, 26.8, 22.2, 20.2, 20.0, 17.9, 15.1, 9.3. HRFABMS $m / z 368.2626\left(\mathrm{M}+\mathrm{H}^{+}, \mathrm{C}_{21} \mathrm{H}_{38} \mathrm{NO}_{2} \mathrm{~S}^{+}\right.$requires 368.2623.

Sample procedure for acidic hydrolysis: Synthesis of $(R)$-2-Benzyl-2-methylbutyric acid (10a): A solution of [2R,4S]-((2R)-2-Ethyl-2-methyl-2,3-dihydrocinnamoyl)-4-isopropyl-2(4-phenyl-3-thiapropyl)-oxazolidine (9a) (240 mg, .64 mmol, 1.0 equiv) in $p$-dioxane (3 mL) and aqueous sulfuric acid solution $(5.3 \mathrm{M}, 3 \mathrm{~mL})$ was heated at reflux for $8 \mathrm{~h}$, cooled to $23{ }^{\circ} \mathrm{C}$ and extracted with diethyl ether $(3 \times 20 \mathrm{~mL})$. The combined organic phases were dried over anhydrous sodium sulfate, filtered and concentrated in vacuo. The residue was purified by distillation under vacuum $\left(150^{\circ} \mathrm{C}, 0.5 \mathrm{mmHg}\right)$ to afford $(R)$-2-benzyl-2-methylbutyric acid (10a) (106 mg, $.52 \mathrm{mmol})$ in 81\% yield as a colourless oil. ${ }^{1} \mathrm{H} \mathrm{NMR}\left(\mathrm{CDCl}_{3}\right) \delta, 11.42$ (bs, 1H), 7.14$7.36(\mathrm{~m}, 5 \mathrm{H}), 3.08(\mathrm{~d}, 1 \mathrm{H}, J=13.3 \mathrm{~Hz}), 2.77(\mathrm{~d}, 1 \mathrm{H}, J=13.3 \mathrm{~Hz}), 1.84(\mathrm{~m}, 1 \mathrm{H}), 1.53(\mathrm{~m}, 1 \mathrm{H})$ $1.13(\mathrm{~s}, 3 \mathrm{H}), 0.98(\mathrm{t}, 3 \mathrm{H}, J=7.4 \mathrm{~Hz}) ;{ }^{13} \mathrm{C} \mathrm{NMR}\left(\mathrm{CDCl}_{3}\right) \delta 183.6,137.6,130.7,128.2,126.7$, $46.5,41.4,32.1,20.8,18.2,15.0,9.3 .[\alpha]^{25}=-7.4^{\circ}\left(\mathrm{c}=26.0, \mathrm{CH}_{2} \mathrm{Cl}_{2}\right)$. The product was 
determined to have 93:7 dr by chiral HPLC analysis (Chiralcel OD column, eluting with 1\% isopropanol $+0.01 \%$ trifluoroacetic acid in hexanes at $0.7 \mathrm{ml} /$ minute) $\mathrm{R}_{\mathrm{t}}=11.78$ minutes (minor enantiomer), 12.32 minutes (major enantiomer). HRMS calc. for $\mathrm{C}_{12} \mathrm{H}_{16} \mathrm{O}_{2}: 192.1150$. Found: $192.1147 \pm 0.0005$.

(R)-2-Ethyl-2-methylpentanoic acid (10b): The reaction was carried out according to the general procedure above starting from $9 \mathbf{b}(252 \mathrm{mg}, .73 \mathrm{mmol})$ to afford $(R)$-2-ethyl-2methylpentanoic acid 10b (83 mg, $.58 \mathrm{mmol})$ in $79 \%$ yield as a colourless oil. ${ }^{1} \mathrm{H} \mathrm{NMR}\left(\mathrm{CDCl}_{3}\right)$ $\delta, 9.81(\mathrm{bs}, 1 \mathrm{H}), 1.22-1.73(\mathrm{~m}, 6 \mathrm{H}), 1.12(\mathrm{~s}, 3 \mathrm{H}), 0.91(\mathrm{t}, 3 \mathrm{H}, J=7.2 \mathrm{~Hz}), 0.87$ (t, 3H, $J=7.4$ $\mathrm{Hz}) ;{ }^{13} \mathrm{C} \mathrm{NMR}\left(\mathrm{CDCl}_{3}\right) \delta 184.6,46.5,41.4,32.1,20.8,18.2,15.0,9.3 .[\alpha]^{25}=+16.4^{\mathrm{o}}(\mathrm{c}=20.0$, EtOH), lit. ${ }^{1}[\alpha]^{20}{ }^{2}=+19.7^{\circ}$ (c=1, EtOH). The product was determined to have 99:1 dr by GC chiral analysis of the corresponding benzyl ester (Chirasil Dex column, He carrier gaz, 14 psi, oven temperature $=130{ }^{\circ} \mathrm{C}, \mathrm{Rt}=13.13$ minutes (major enantiomer), 15.97 minutes (minor enantiomer). HRFABMS $m / z\left(\mathrm{M}+\mathrm{H}, 145.1232 \mathrm{C}_{8} \mathrm{H}_{16} \mathrm{O}_{2}{ }^{+}\right.$requires 145.1228$)$.

(S)-6-Chloro-2-ethyl-2-methylhexanoic acid (10j): The reaction was carried out according to the general procedure above starting from $9 \mathbf{i}(200 \mathrm{mg}, .45 \mathrm{mmol})$ to afford $(S)-6$ Chloro-2-ethyl-2-methyl-hexanoic acid $\mathbf{1 0 j}(69 \mathrm{mg}, .36 \mathrm{mmol})$ in $81 \%$ yield as a colourless oil. ${ }^{1} \mathrm{H}$ NMR $\left(\mathrm{CDCl}_{3}\right) \delta, 11.12(\mathrm{bs}, 1 \mathrm{H}), 3.53(\mathrm{t}, 2 \mathrm{H}, J=6.7 \mathrm{~Hz}), 1.67-1.36(\mathrm{~m}, 8 \mathrm{H}), 1.15(\mathrm{~s}, 3 \mathrm{H})$, $0.88(\mathrm{t}, 3 \mathrm{H}, J=6.5 \mathrm{~Hz}) ;{ }^{13} \mathrm{C} \mathrm{NMR}\left(\mathrm{CDCl}_{3}\right) \delta 183.8,46.4,45.1,38.0,33.3,32.1,22.3,20.9,9.3$. $[\alpha]^{25}=-13.2^{\circ}\left(\mathrm{c}=10.0, \mathrm{CH}_{2} \mathrm{Cl}_{2}\right)$. The product was determined to have 95:5 dr by chiral GC (Chirasil Dex column, He carrier gas, $25 \mathrm{psi}$, oven temperature $=100{ }^{\circ} \mathrm{C}$ ), $\mathrm{Rt}=6.76$ minutes (minor enantiomer), 10.75 minutes (major enantiomer). HRFABMS $m / z 193.1000\left(\mathrm{M}+\mathrm{H}^{+}\right.$, $\mathrm{C}_{9} \mathrm{H}_{18} \mathrm{ClO}_{2}^{+}$requires 193.0995).

' G. Fronza, G. Fogliato, C. Fuganti, P. Grasselli, R. Rigoni, Tetrahedron 1996, 52, 14281. 
(R)-6-Chloro-2-ethyl-2-methylhexanoic acid (10d): The reaction was carried out according to the general procedure above starting from $9 \mathbf{d}(150 \mathrm{mg}, .34 \mathrm{mmol})$ to afford $(R)-6-$ Chloro-2-ethyl-2-methyl-hexanoic acid $\mathbf{1 0 d}(53 \mathrm{mg}, .27 \mathrm{mmol})$ in $80 \%$ yield as a colourless oil. The product had identical spectral characteristics to $\mathbf{1 0} \mathbf{j}$ but displayed opposite optical rotation and chiral GC behaviour. $[\alpha]^{25}=+14.8^{\circ}\left(\mathrm{c}=3.35, \mathrm{CH}_{2} \mathrm{Cl}_{2}\right)$. The product was determined to have 95:5 dr by chiral GC (Chirasil Dex column, He carrier gas, $25 \mathrm{psi}$, oven temperature $=100{ }^{\circ} \mathrm{C}$ ), $\mathrm{Rt}=6.67$ minutes (major enantiomer), 10.68 minutes (minor enantiomer).

(S)-2-Benzyl-2-methylbutyric acid (10g): The reaction was carried out according to the general procedure above starting from $9 \mathrm{~g}(234 \mathrm{mg}, .53 \mathrm{mmol})$ to afford $(S)$-2-benzyl-2methylbutyric acid $(\mathbf{1 0 g})(81 \mathrm{mg}, .42 \mathrm{mmol})$ in $60 \%$ yield as a colourless oil (the product contained residual diethyl ether and dioxane which were not removed due to volatility of the product). The product had identical spectral characteristics to $10 \mathrm{a}$ but displayed opposite optical rotation and chiral GC behaviour. $[\alpha]^{25}=+8.5^{\circ}\left(\mathrm{c}=35.0, \mathrm{CH}_{2} \mathrm{Cl}_{2}\right)$. The product was determined to have 93:7 dr by chiral HPLC analysis (Chiralcel OD column, eluting with 1\% isopropanol + $0.01 \%$ trifluoroacetic acid in hexanes at $0.7 \mathrm{ml} /$ minute) $\mathrm{R}_{\mathrm{t}}=11.78$ minutes (major enantiomer), 12.32 minutes (minor enantiomer).

(S)-2-Ethyl-2-methylpentanoic acid (10h): The reaction was carried out according to the general procedure above starting from $9 \mathbf{h}(239 \mathrm{mg}, .70 \mathrm{mmol})$ to afford $(S)$-2-ethyl-2methylpentanoic acid (10h) $(81 \mathrm{mg}, .57 \mathrm{mmol})$ in $82 \%$ yield as a colourless oil. The product had identical spectral characteristics to $\mathbf{1 0 b}$ but displayed opposite optical rotation and chiral GC behaviour. $[\alpha]^{25}=-18.0^{\circ}(\mathrm{c}=10.5, \mathrm{EtOH})$, lit. $^{1}$ (for enantiomer 10b) $[\alpha]^{20}{ }_{\mathrm{D}}=+19.0^{\circ}(\mathrm{c}=1, \mathrm{EtOH})$. The product was determined to have 99:1 dr by GC chiral analysis of the corresponding benzyl 
ester (Chirasil Dex column, He carrier gaz, 14 psi, oven temperature $=130{ }^{\circ} \mathrm{C}, \mathrm{Rt}=13.13$ minutes (minor enantiomer), 15.97 minutes (major enantiomer).

Sample procedure for reduction to the primary alcohol with lithium

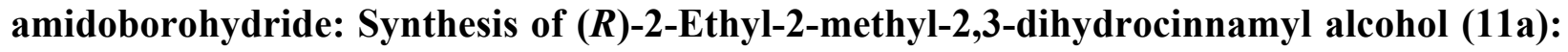

A solution of $n$-butyllithium in hexanes $(2.50 \mathrm{M}, 813 \mu \mathrm{L}, 1.87 \mathrm{mmol}, 3.9$ equiv.) was slowly added to a stirred solution of diisopropylamine $(275 \mu \mathrm{L}, 1.96 \mathrm{mmol}, 4.1$ equiv.) in tetrahydrofuran $(2.0 \mathrm{~mL})$ at $0{ }^{\circ} \mathrm{C}$. After stirring for 10 minutes, borane-ammonia complex $(90 \%$, $60 \mathrm{mg}, 1.9 \mathrm{mmol}, 4.0$ equiv.) was added in one portion. After stirring at $0{ }^{\circ} \mathrm{C}$ for 15 minutes the mixture was warmed to $23{ }^{\circ} \mathrm{C}$ and a solution of $[2 R, 4 S]-((2 R)-2$-Ethyl-2-methyl-2,3dihydrocinnamoyl)-4-isopropyl-2-(4-phenyl-3-thiapropyl)-oxazolidine 9a (210 mg, .48 mmol, 1.0 equiv.) in tetrahydrofuran $(5 \mathrm{~mL})$ was added via cannula. The mixture was heated at reflux for 24 hours, then cooled to $0{ }^{\circ} \mathrm{C}$ and quenched with aqueous hydrochloric acid $(3 \mathrm{M}, 5 \mathrm{~mL})$. The resulting mixture was warmed to $23{ }^{\circ} \mathrm{C}$ and stirred for 30 minutes, at which point aqueous sodium hydroxide $(3 \mathrm{M}, 10 \mathrm{~mL})$ was added. The mixture was stirred at $23{ }^{\circ} \mathrm{C}$ for 30 minutes and extracted with diethyl ether $(3 \times 20 \mathrm{~mL})$. The combined organic layers were dried over anhydrous sodium sulfate, filtered and concentrated in vacuo. Column chromatography on silica gel eluting with $30 \%$ diethyl ether in hexanes afforded 11 a $(72 \mathrm{mg}, .41 \mathrm{mmol}$,) in $85 \%$ yield as a colorless oil. ${ }^{1} \mathrm{H} \mathrm{NMR}\left(\mathrm{CDCl}_{3}\right) \delta, 7.17-7.27(\mathrm{~m}, 5 \mathrm{H}), 3.34(\mathrm{~d}, 1 \mathrm{H}, J=11.0 \mathrm{~Hz}), 3.32(\mathrm{~d}, 1 \mathrm{H}, J=$ $10.6 \mathrm{~Hz}), 2.63(\mathrm{~d}, 1 \mathrm{H}, J=13.3 \mathrm{~Hz}), 2.58(\mathrm{~d}, 1 \mathrm{H}, J=12.9 \mathrm{~Hz}), 1.28-1.42(\mathrm{~m}, 3 \mathrm{H}), 0.93(\mathrm{t}, 3 \mathrm{H}, J=$ $7.4 \mathrm{~Hz}), 0.83(\mathrm{~s}, 3 \mathrm{H}) ;{ }^{13} \mathrm{C} \mathrm{NMR}\left(\mathrm{CDCl}_{3}\right) \delta 138.9,130.7,128.1,126.1,68.5,42.9,39.2,29.3$, 21.2, 8.5. $[\alpha]^{25}{ }_{\mathrm{D}}=+8.0^{\circ}\left(\mathrm{c}=69.5, \mathrm{CH}_{2} \mathrm{Cl}_{2}\right)$. The product was determined to have $93: 7 \mathrm{dr}$ by chiral HPLC analysis (Chiralcel OD column, eluting with 1\% isopropanol in hexanes at $0.7 \mathrm{ml} / \mathrm{minute}$ - 
$\mathrm{R}_{\mathrm{t}}=21.1$ minutes (major enantiomer), 23.7 minutes (minor enantiomer). HRMS calc. for $\mathrm{C}_{12} \mathrm{H}_{18} \mathrm{O}: 178.1358$. Found: $178.1362 \pm 0.0005$.

(S)-2-Ethyl-2-propyl-pent-4-en-1-ol (11k): The reaction was carried out according to the general procedure above starting from $9 k$ ( $40 \mathrm{mg}, .11 \mathrm{mmol})$ to afford $(S)$-2-Ethyl-2-propylpent-4-en-1-ol $11 \mathbf{k}(15 \mathrm{mg}, .09 \mathrm{mmol})$ in 84\%yield as a colorless oil: ${ }^{1} \mathrm{H} \mathrm{NMR}\left(\mathrm{CDCl}_{3}\right) \delta, 5.84$ $(\mathrm{m}, 1 \mathrm{H}), 5.04(\mathrm{~m}, 2 \mathrm{H}), 3.38(\mathrm{~s}, 2 \mathrm{H}), 2.02(\mathrm{~d}, 2 \mathrm{H}, J=7.4 \mathrm{~Hz}), 1.33-1.17(\mathrm{~m}, 7 \mathrm{H}), 0.90(\mathrm{t}, 3 \mathrm{H}, J=$ $7.1 \mathrm{~Hz}), 0.83(\mathrm{t}, 3 \mathrm{H}, J=7.8 \mathrm{~Hz}) ;{ }^{13} \mathrm{C} \mathrm{NMR}\left(\mathrm{CDCl}_{3}\right) \delta 135.5,117.2,67.4,40.6,39.3,36.1,26.3$, 16.6, 15.4, 7.9. $[\alpha]^{25}=-15.1^{\circ}\left(\mathrm{c}=8.6, \mathrm{CH}_{2} \mathrm{Cl}_{2}\right)$. The product was determined to have $96: 4 \mathrm{dr}$ by chiral GC analysis (Chirasil Dex column, He carrier gas, 14 psi, oven temperature $=80{ }^{\circ} \mathrm{C}$ ) $\mathrm{R}_{\mathrm{t}}=$ 14.18 minutes (major enantiomer), 16.18 minutes (minor enantiomer). HRFABMS $\mathrm{m} / \mathrm{z} 157.1597$ $\left(\mathrm{M}+\mathrm{H}^{+}, \mathrm{C}_{10} \mathrm{H}_{21} \mathrm{O}^{+}\right.$requires 157.1592).

(R)-2-Ethyl-2-propyl-pent-4-en-1-ol (11e): The reaction was carried out according to the general procedure above starting from 9e $(783 \mathrm{mg}, 2.12 \mathrm{mmol})$ to afford $(R)$-2-ethyl-2propyl-pent-4-en-1-ol 11e $(280 \mathrm{mg}, 1.79 \mathrm{mmol})$ in $84 \%$ yield as a colorless oil. The product displayed identical spectral characteristics to $\mathbf{1 1 k}$ but with opposite optical rotation and chiral GC behaviour. $[\alpha]^{25}{ }_{D}=+14.4 .^{\circ}\left(\mathrm{c}=8.0, \mathrm{CH}_{2} \mathrm{Cl}_{2}\right)$. The product was determined to have 95:5 dr by chiral GC analysis (Chirasil Dex column, He carrier gas, 14 psi, oven temperature $=80{ }^{\circ} \mathrm{C}$ ) $\mathrm{R}_{\mathrm{t}}=$ 14.96 minutes (minor enantiomer), 16.53 minutes (major enantiomer).

(S)-2-Ethyl-2-methyl-2,3-dihydrocinnamyl alcohol (11f): The reaction was carried out according to the general procedure above starting from $9 f(39 \mathrm{mg}, .10 \mathrm{mmol})$ to afford $(S)-2$ ethyl-2-methyl-2,3-dihydrocinnamyl alcohol $\mathbf{1 1 f}(17 \mathrm{mg}, .09 \mathrm{mmol})$ in $87 \%$ yield as a colorless oil. The product displayed identical spectral characteristics to 11a but with opposite optical rotation and chiral GC behaviour. $[\alpha]^{25}{ }_{\mathrm{D}}^{=-7.1^{\circ}}\left(\mathrm{c}=69.5, \mathrm{CH}_{2} \mathrm{Cl}_{2}\right)$. The product was determined to 
have 97:3 dr by chiral HPLC analysis (Chiralcel OD column, eluting with 1\% isopropanol in hexanes at $0.7 \mathrm{ml} /$ minute- $\mathrm{R}_{\mathrm{t}}=21.3$ minutes (minor enantiomer), 23.7 minutes (major enantiomer).

(S)-2-Ethyl-2-methyl-2,3-dihydrocinnamyl alcohol (11g): The reaction was carried out according to the general procedure above starting from $9 \mathrm{~g}(212 \mathrm{mg}, .48 \mathrm{mmol})$ to afford $(S)$-2ethyl-2-methyl-2,3-dihydrocinnamyl alcohol $\mathbf{1 1} \mathbf{g}(73 \mathrm{mg}, .41 \mathrm{mmol})$ in $85 \%$ yield as a colorless oil. The product displayed identical spectral characteristics to 11a but with opposite optical rotation and chiral GC behaviour. $[\alpha]^{25}{ }^{2}=-7.1^{\circ}\left(\mathrm{c}=14.2, \mathrm{CH}_{2} \mathrm{Cl}_{2}\right)$. The product was determined to have 93:7 dr by chiral HPLC analysis (Chiralcel OD column, eluting with 1\% isopropanol in hexanes at $0.7 \mathrm{ml} /$ minute- $\mathrm{R}_{\mathrm{t}}=24.4$ minutes (major enantiomer), 22.2 minutes (minor enantiomer). 


\section{Compound 2}

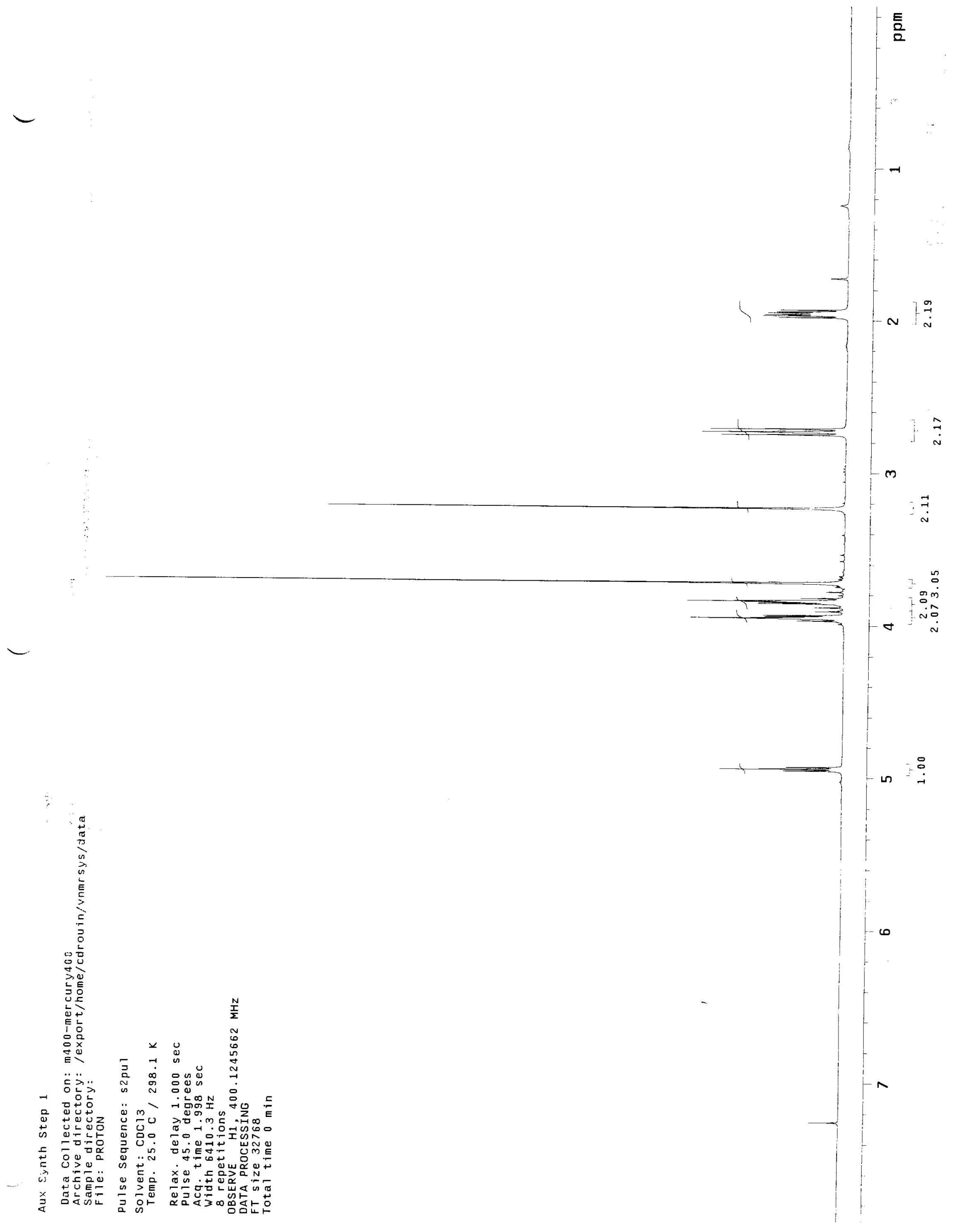


Compound 4

$m$

\}$\stackrel{m}{\circ} r$

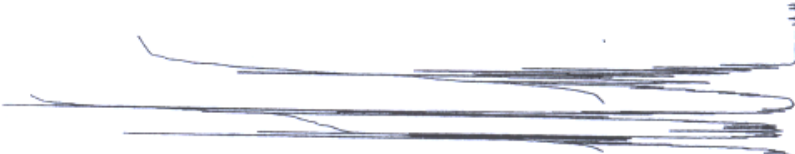

$\left[\begin{array}{l}\overrightarrow{0} \\ 0 \\ 0 \\ 0 \\ 0\end{array}\right.$

$\rightarrow-f^{-1}$

n $]_{0}^{\infty}$

$\omega$

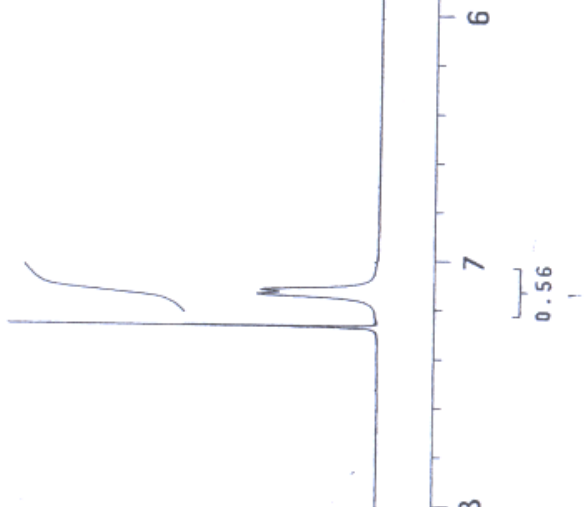

$-\infty$ 
Compound 5
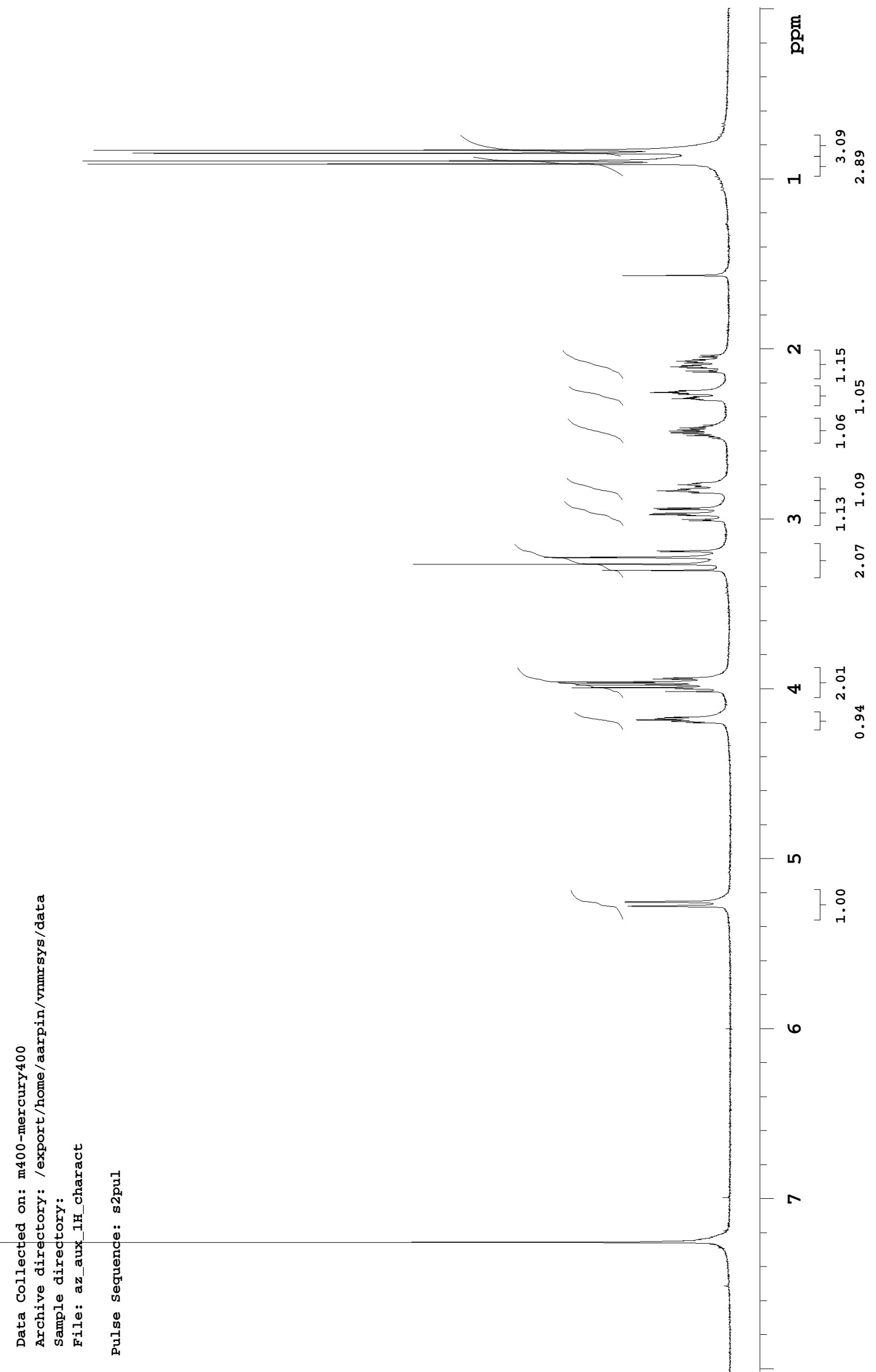
Compound 6a

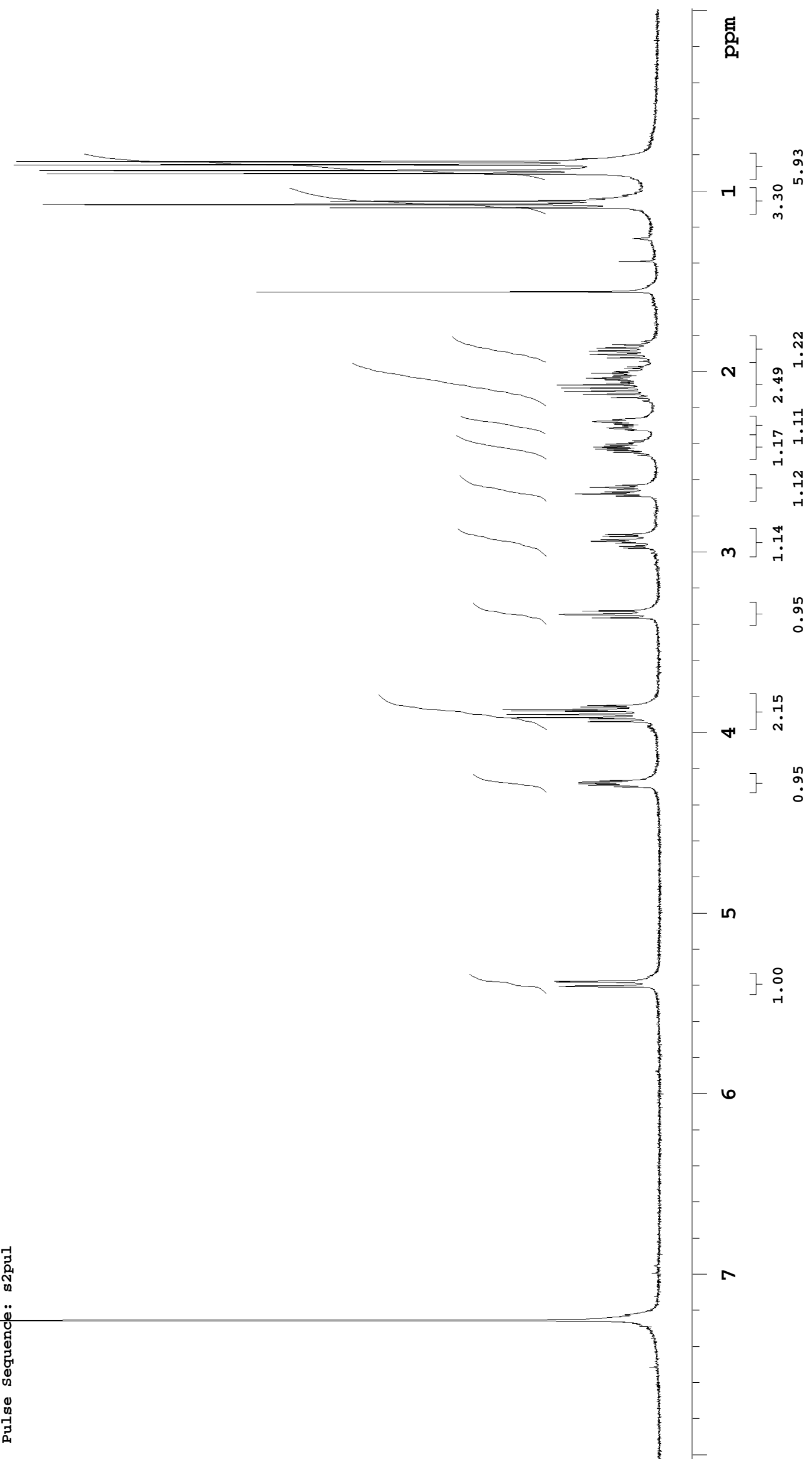


Compound $6 \mathrm{~b}$
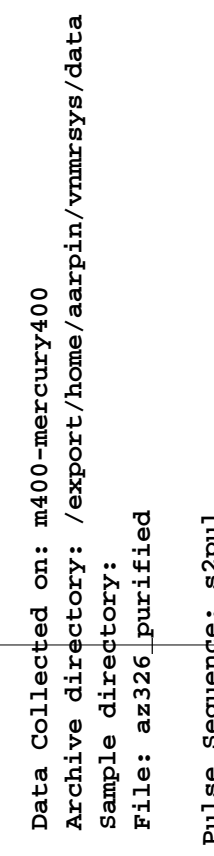
Compound $6 \mathrm{c}$

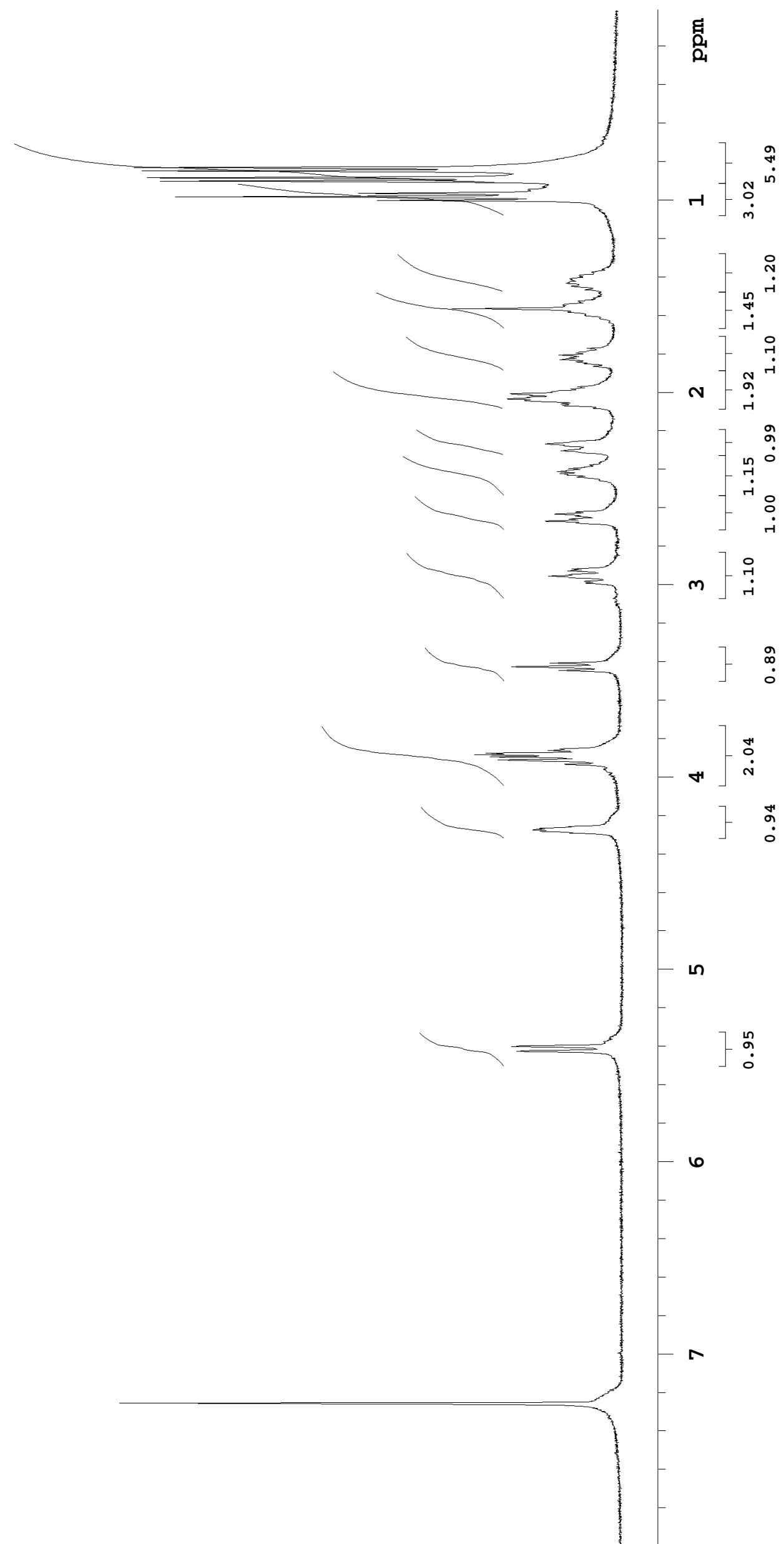


Compound 6d

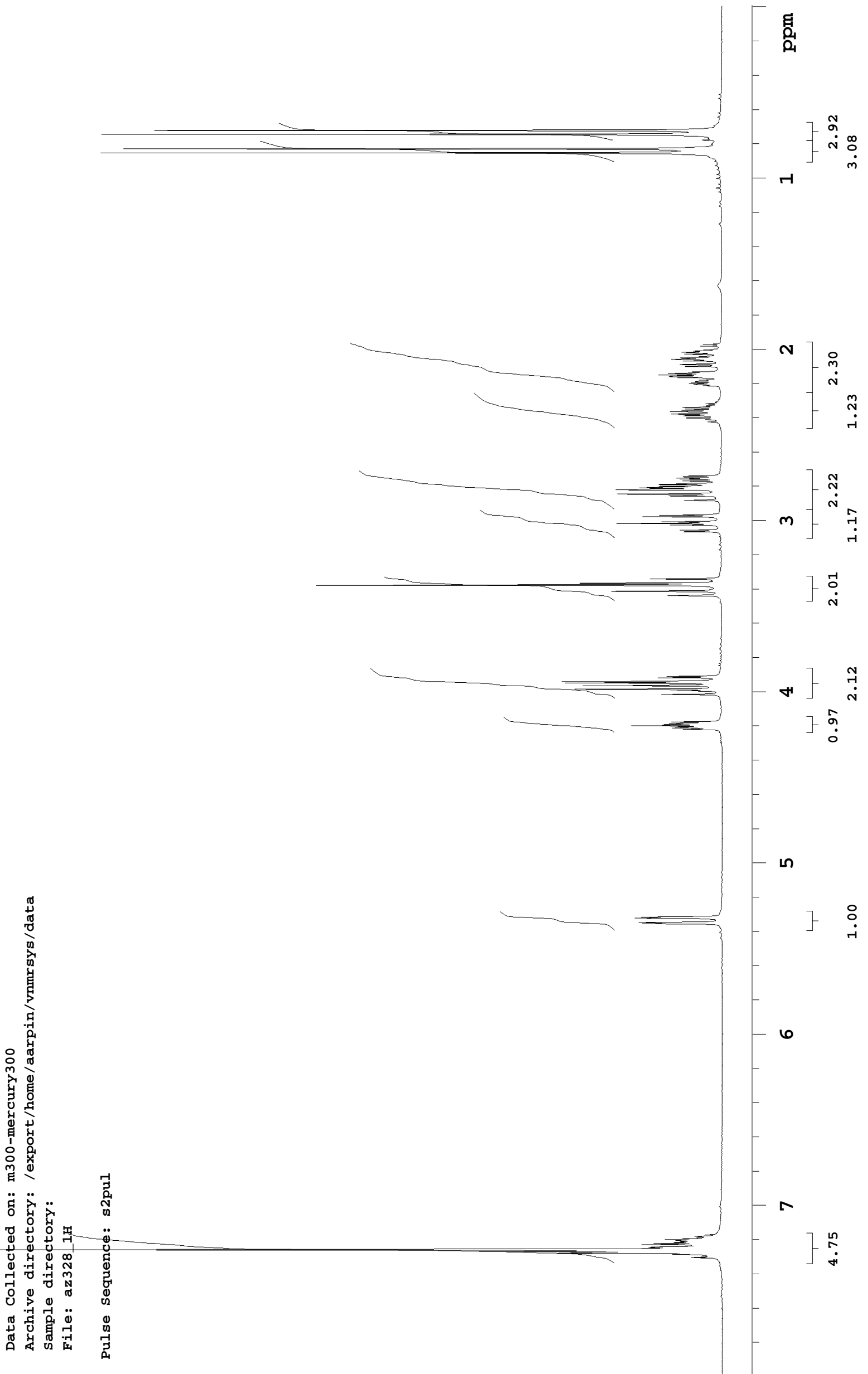


Compound 7a

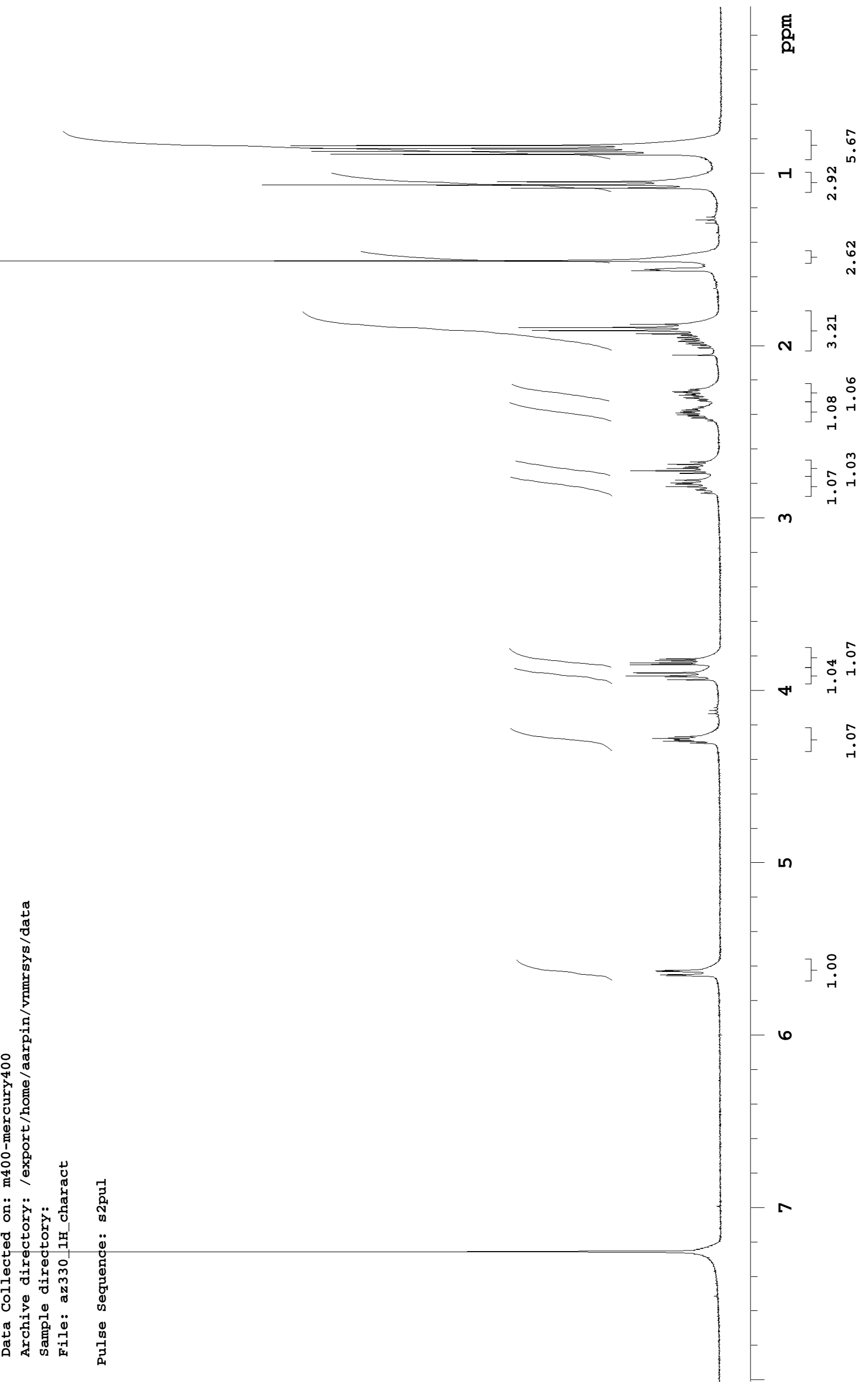


Compound $7 \mathrm{~b}$

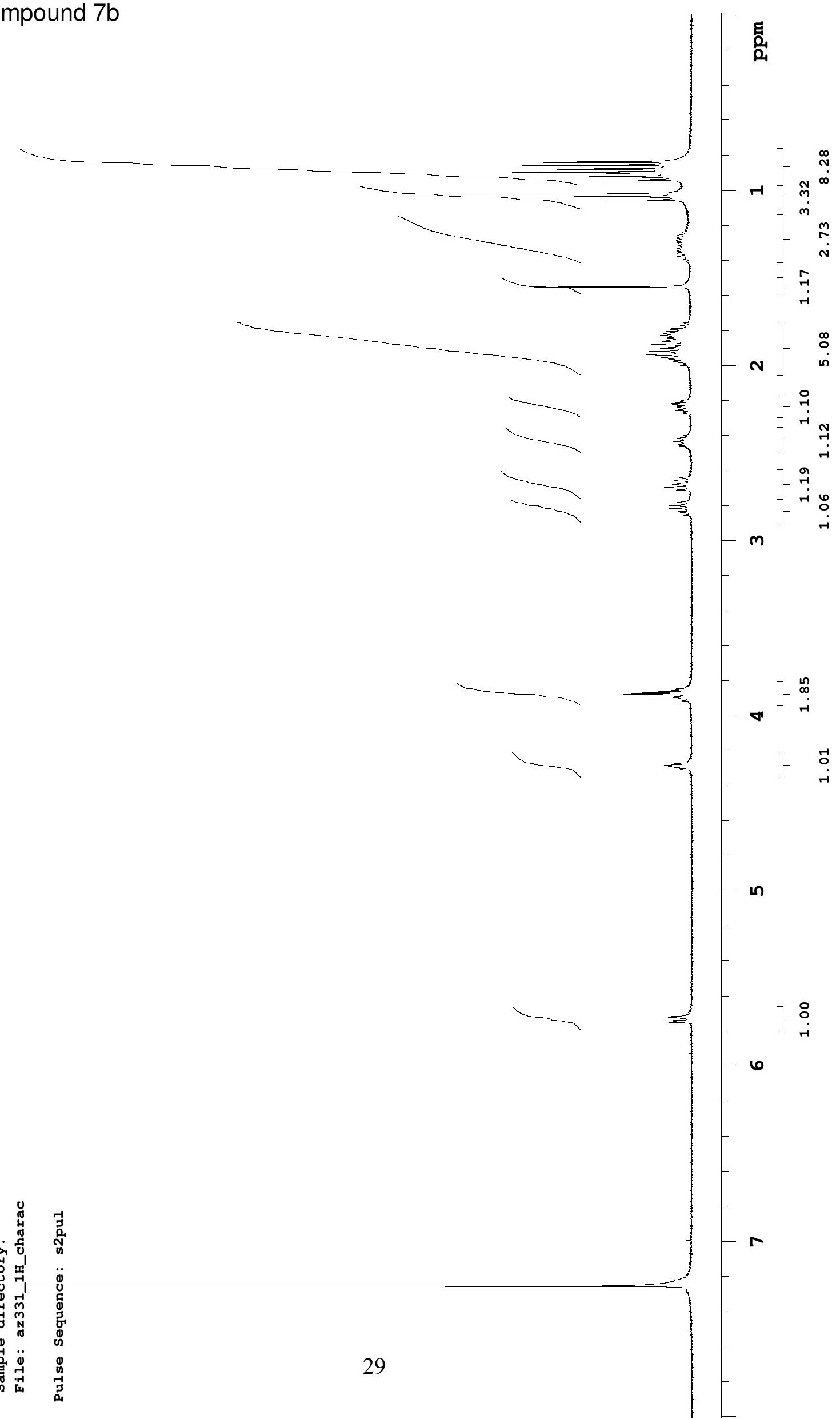


Compound 7c

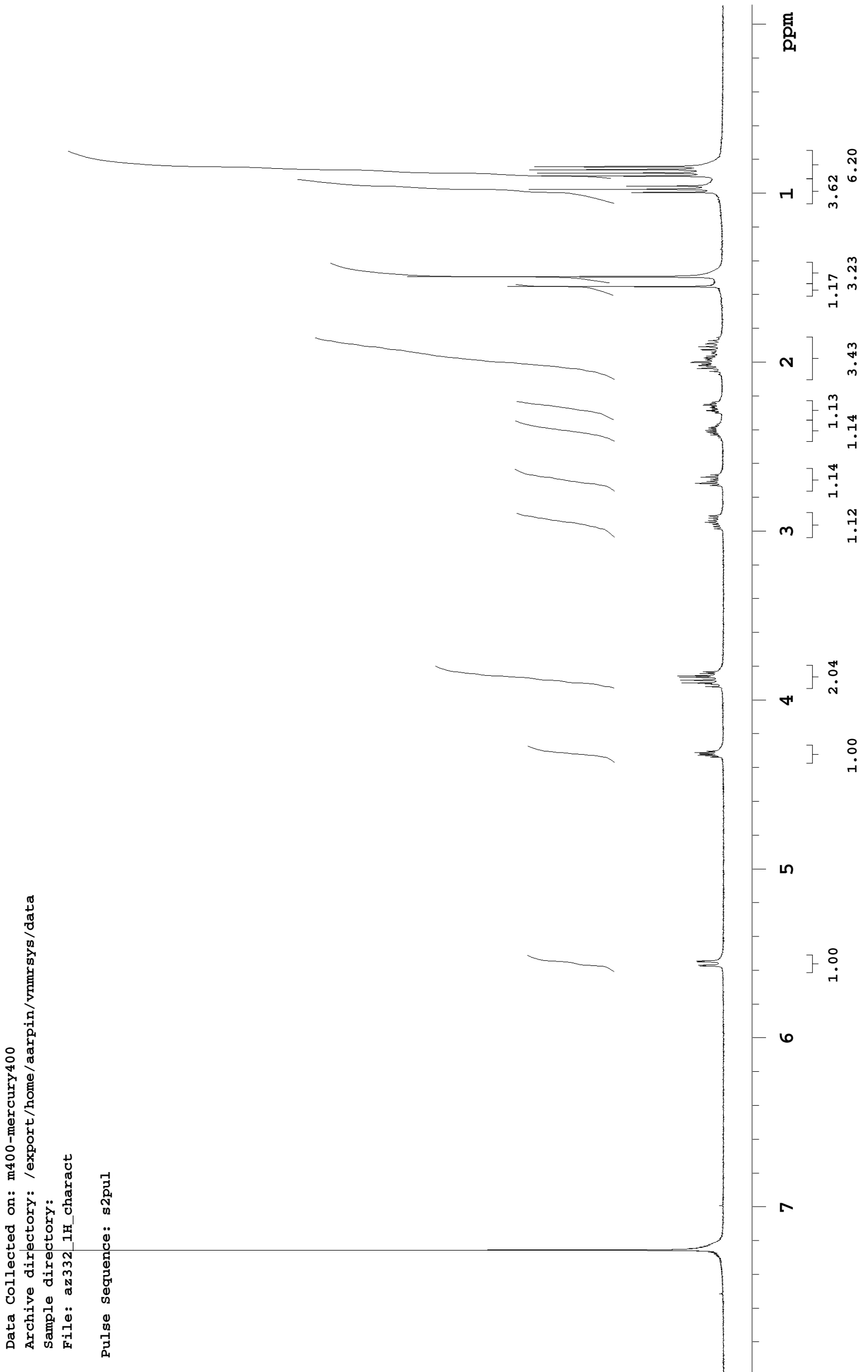


Compound 7d

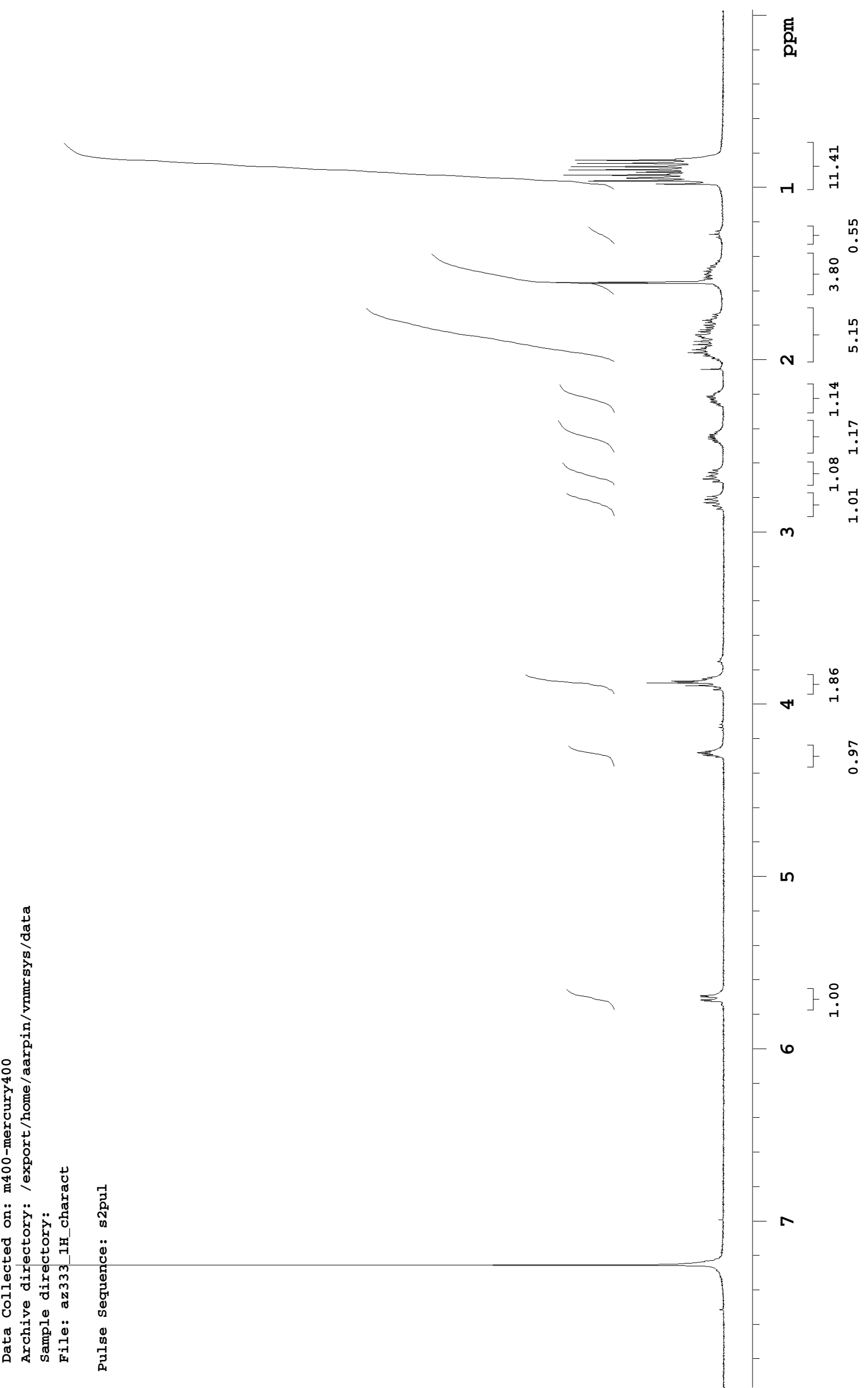


Compound $7 \mathrm{e}$

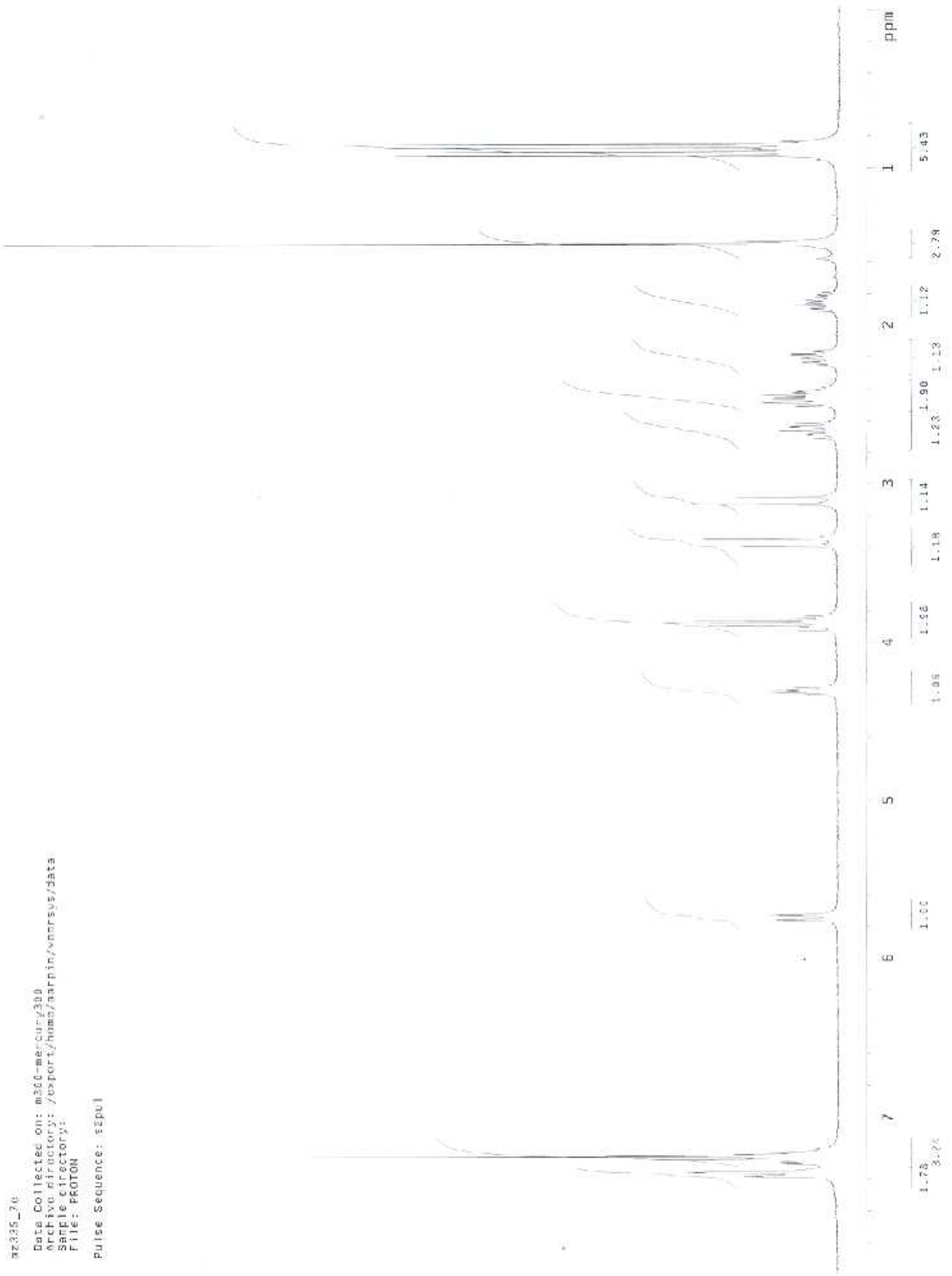


Compound $7 f$

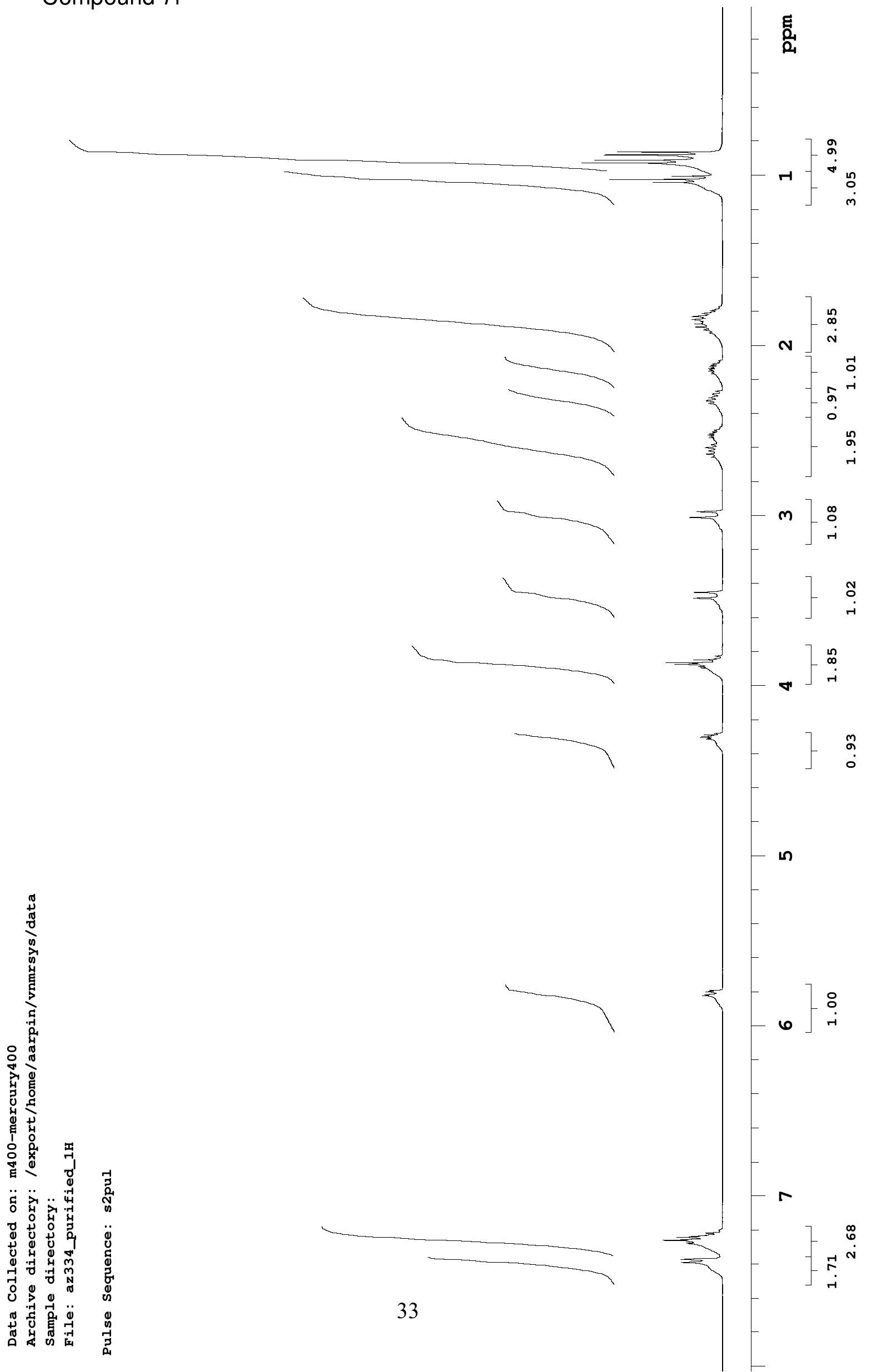


Compound $9 \mathrm{a}$

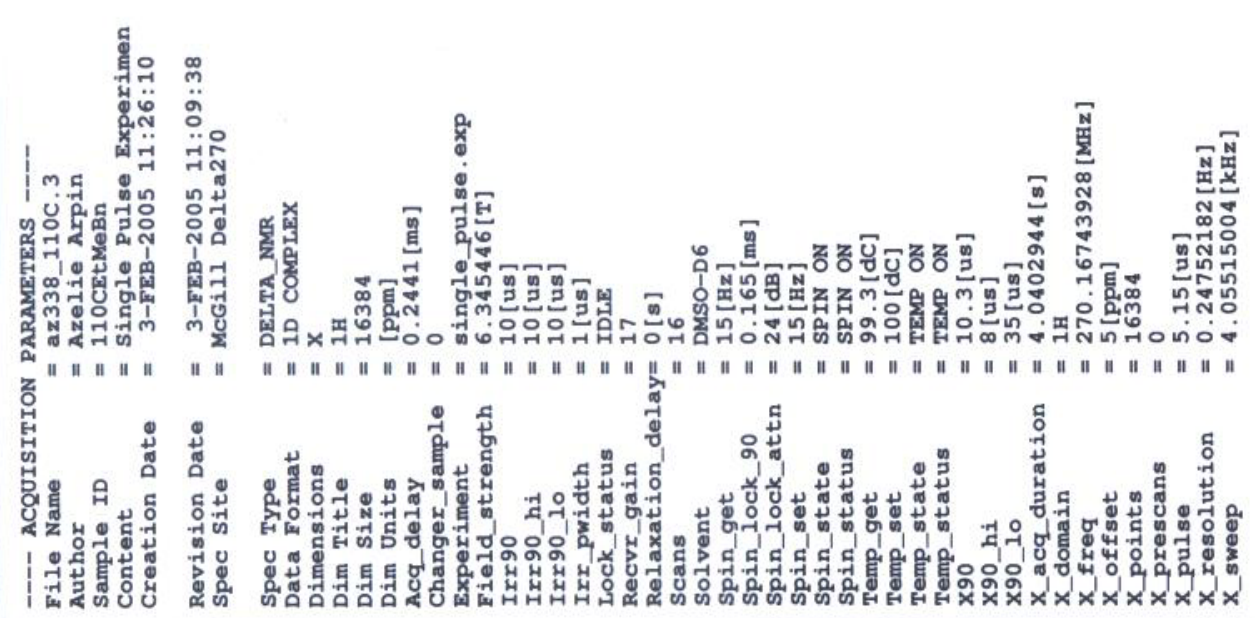


Compound 9b

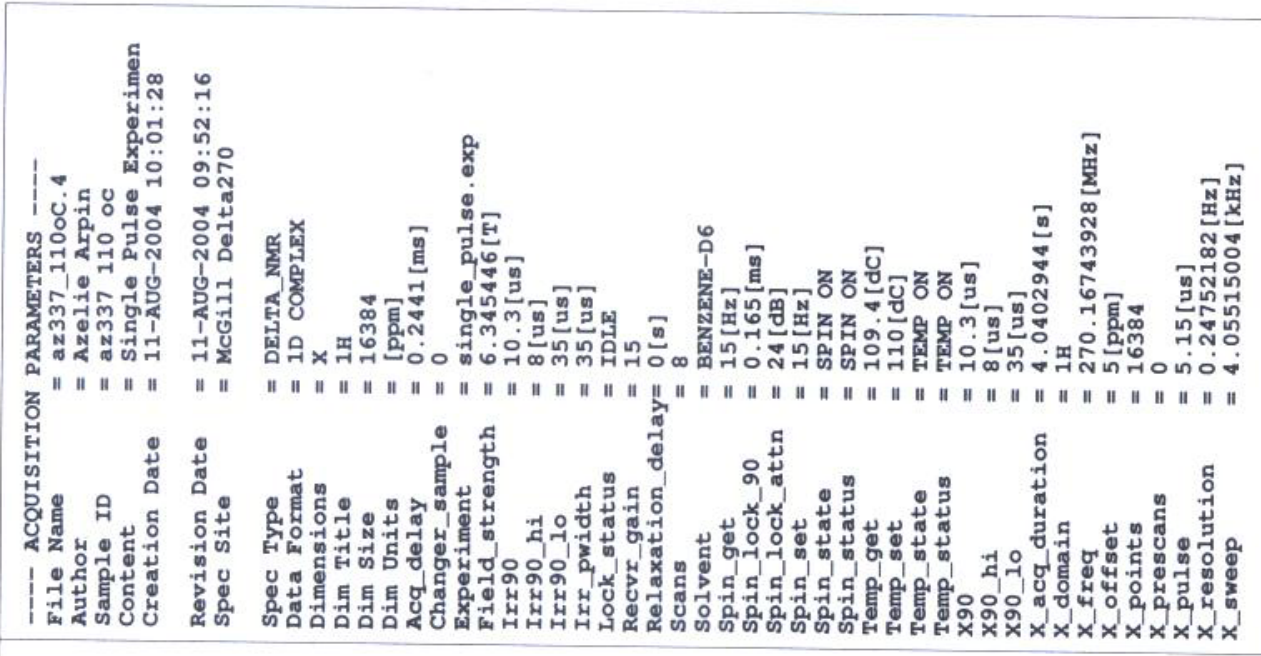

E8EIt*0

LISOI'0

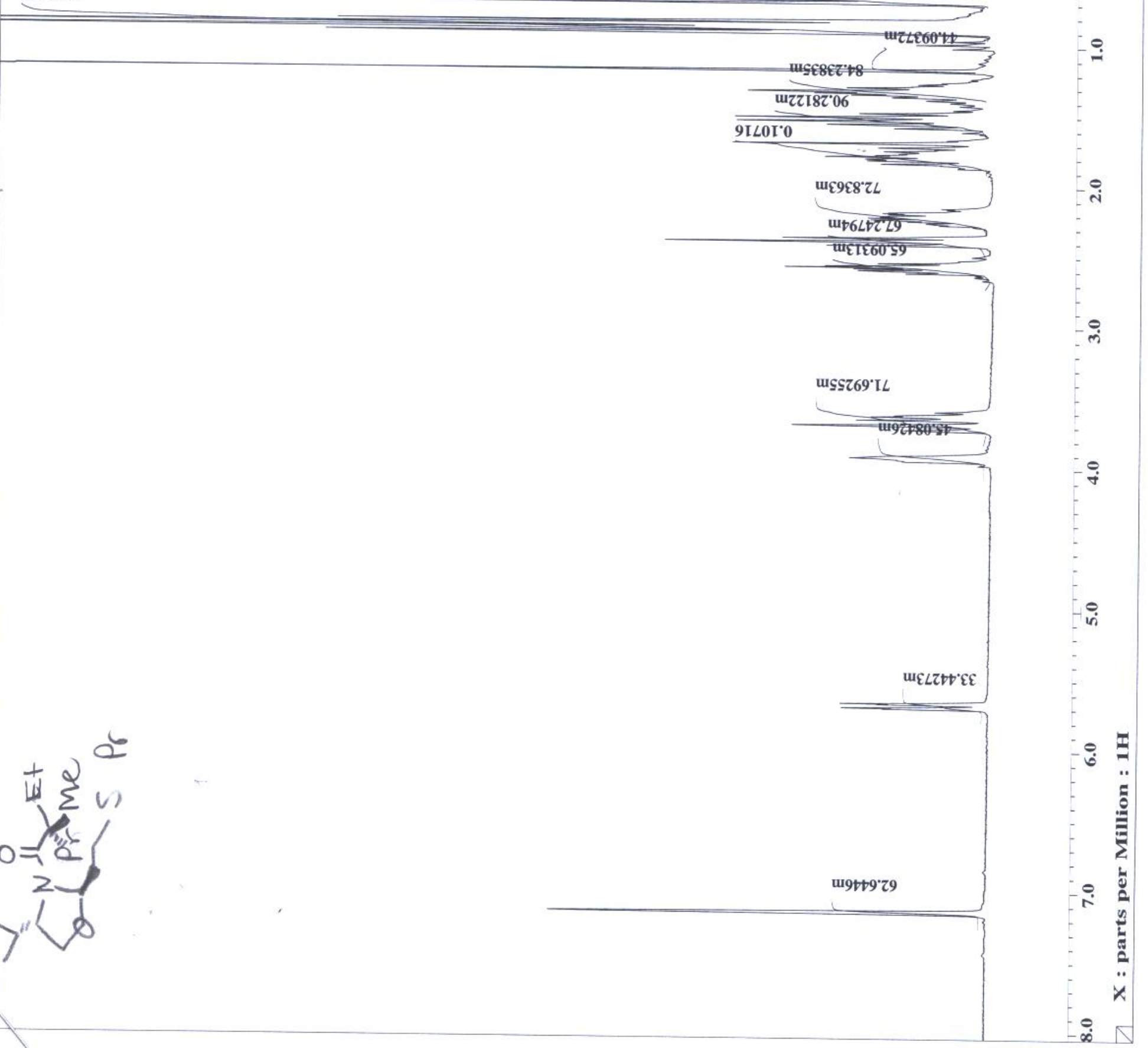


Compound 9c

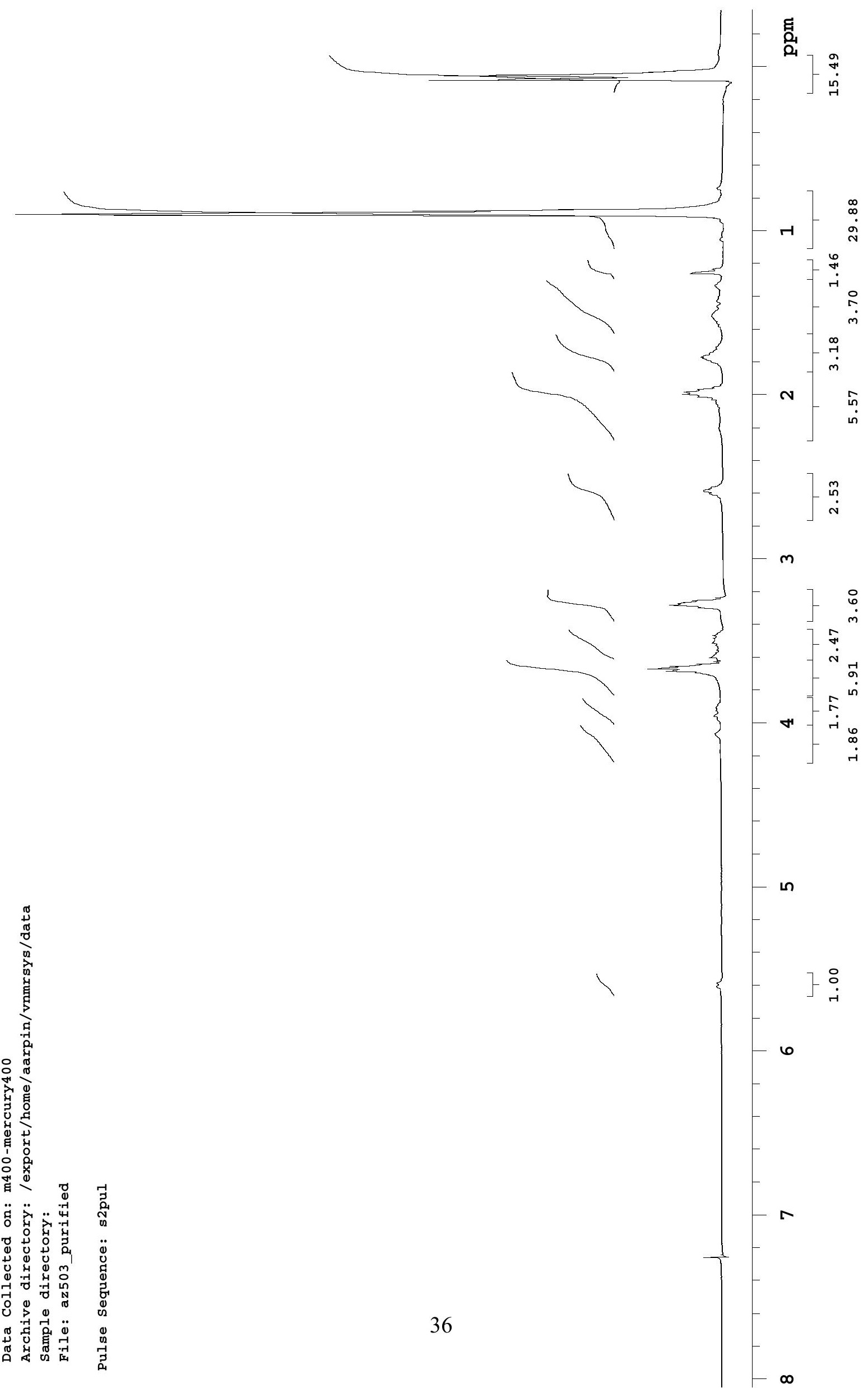


Compound 9d

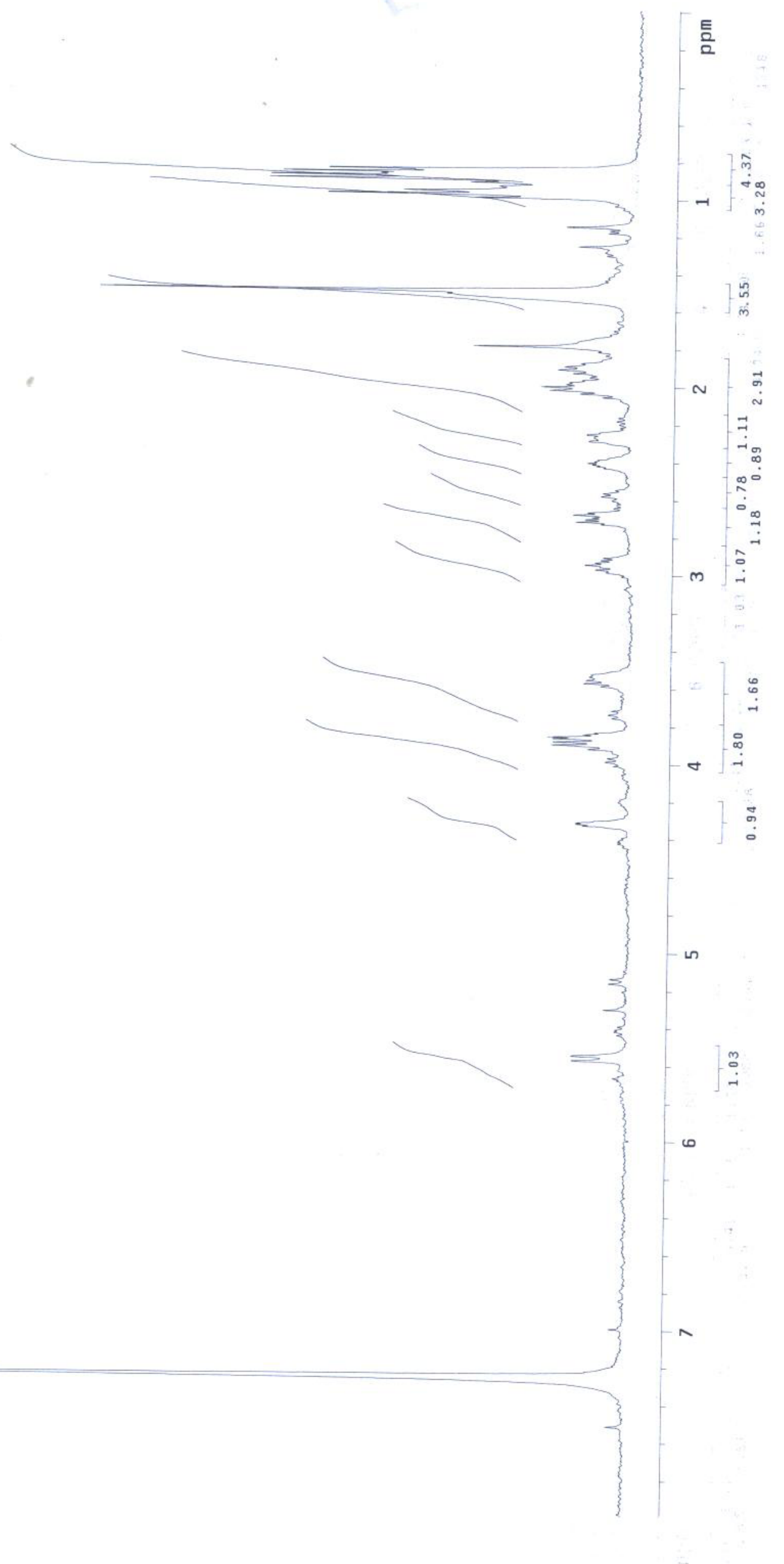


Compound $9 f$

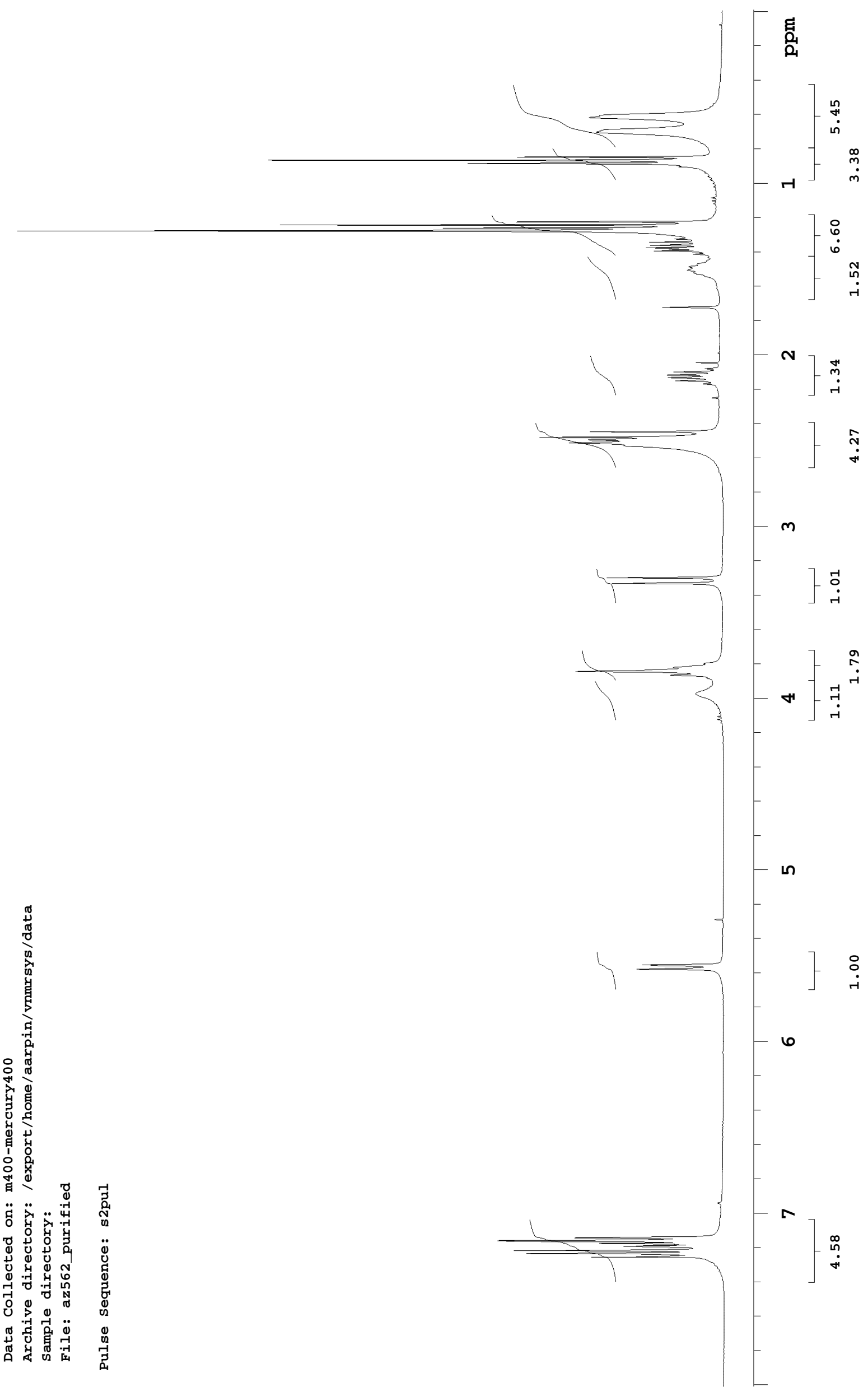


Compound $9 \mathrm{~g}$

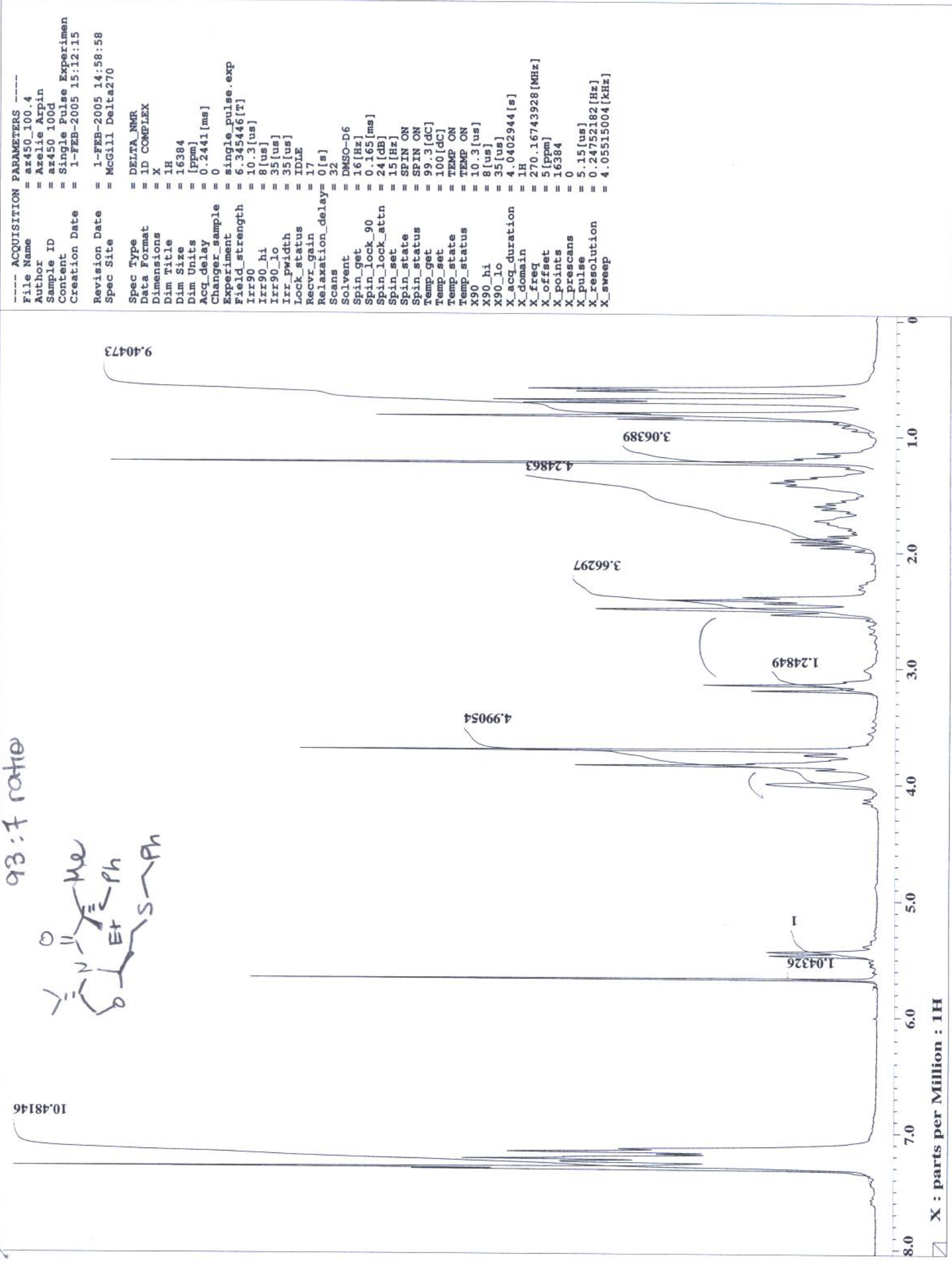




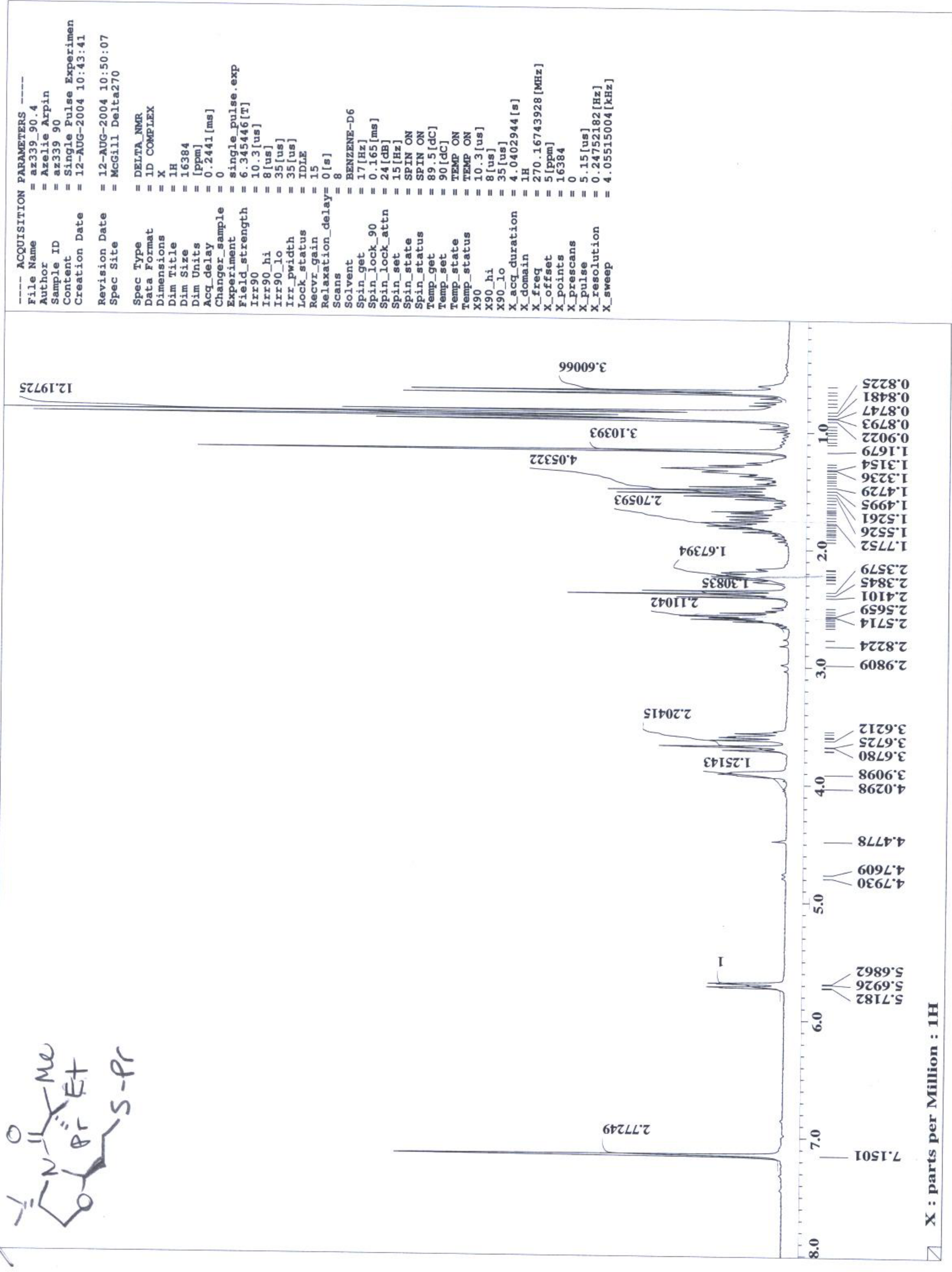


Compound 9i

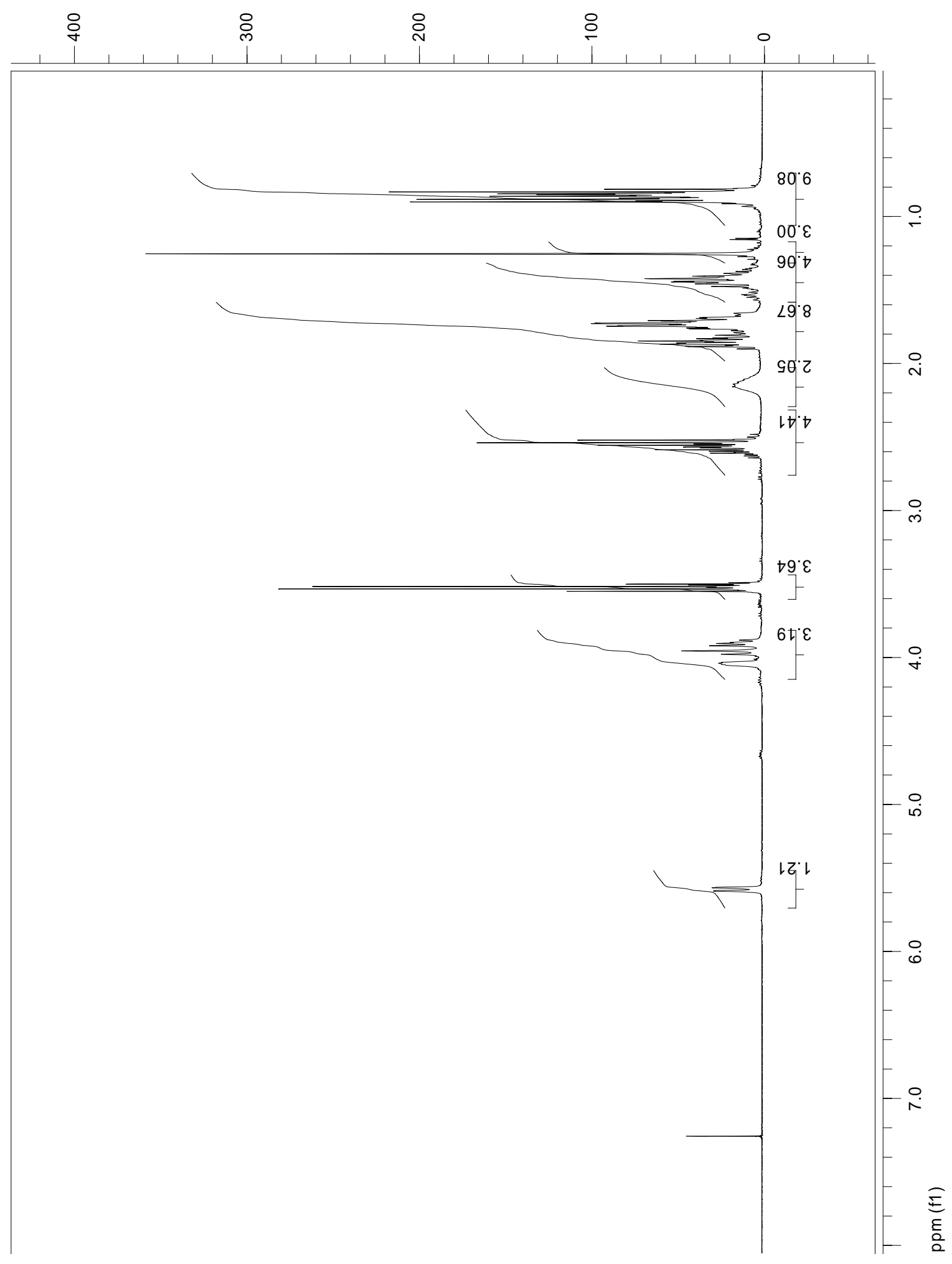


Compound 9j

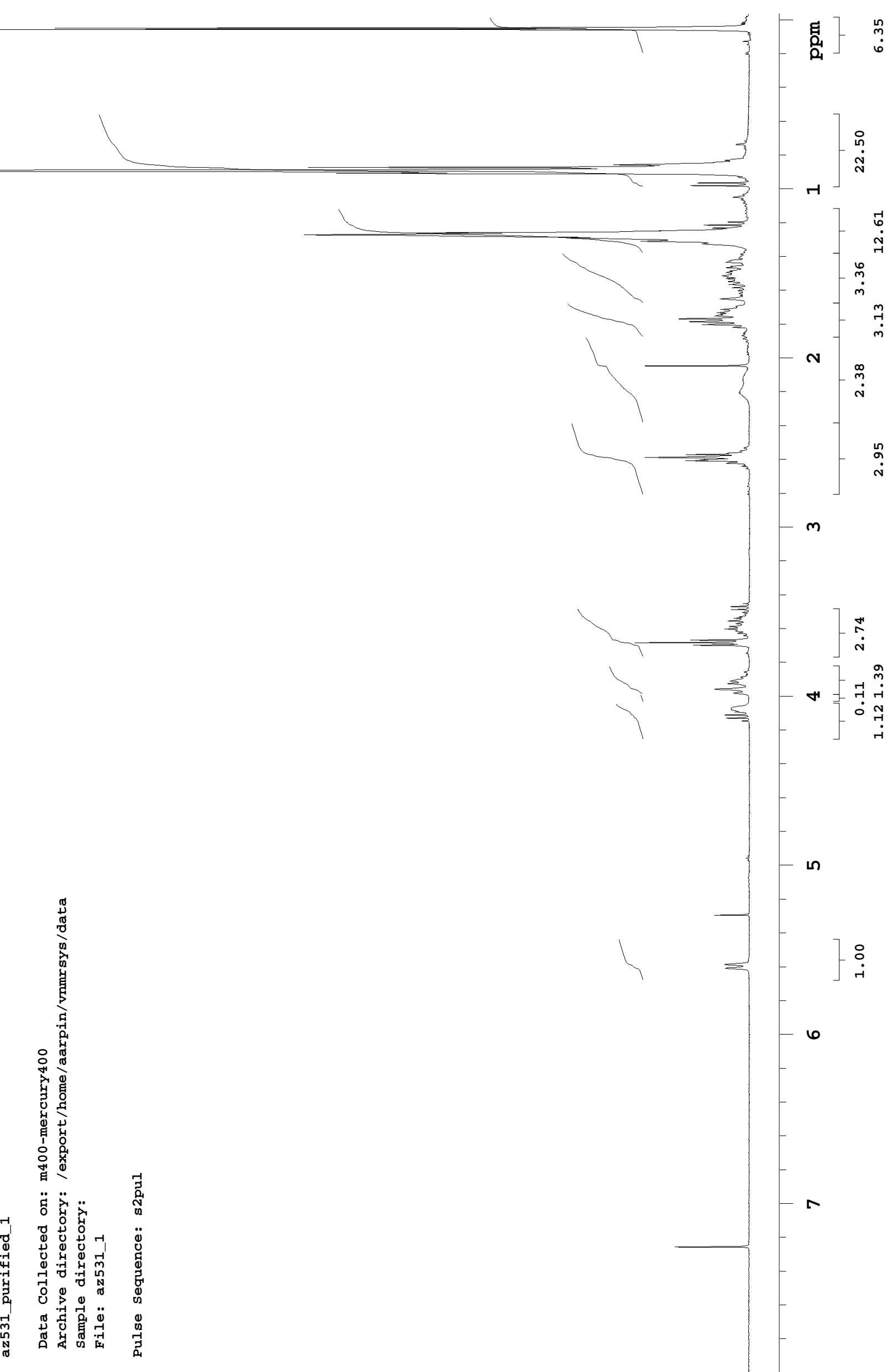


Compound 9k

틀

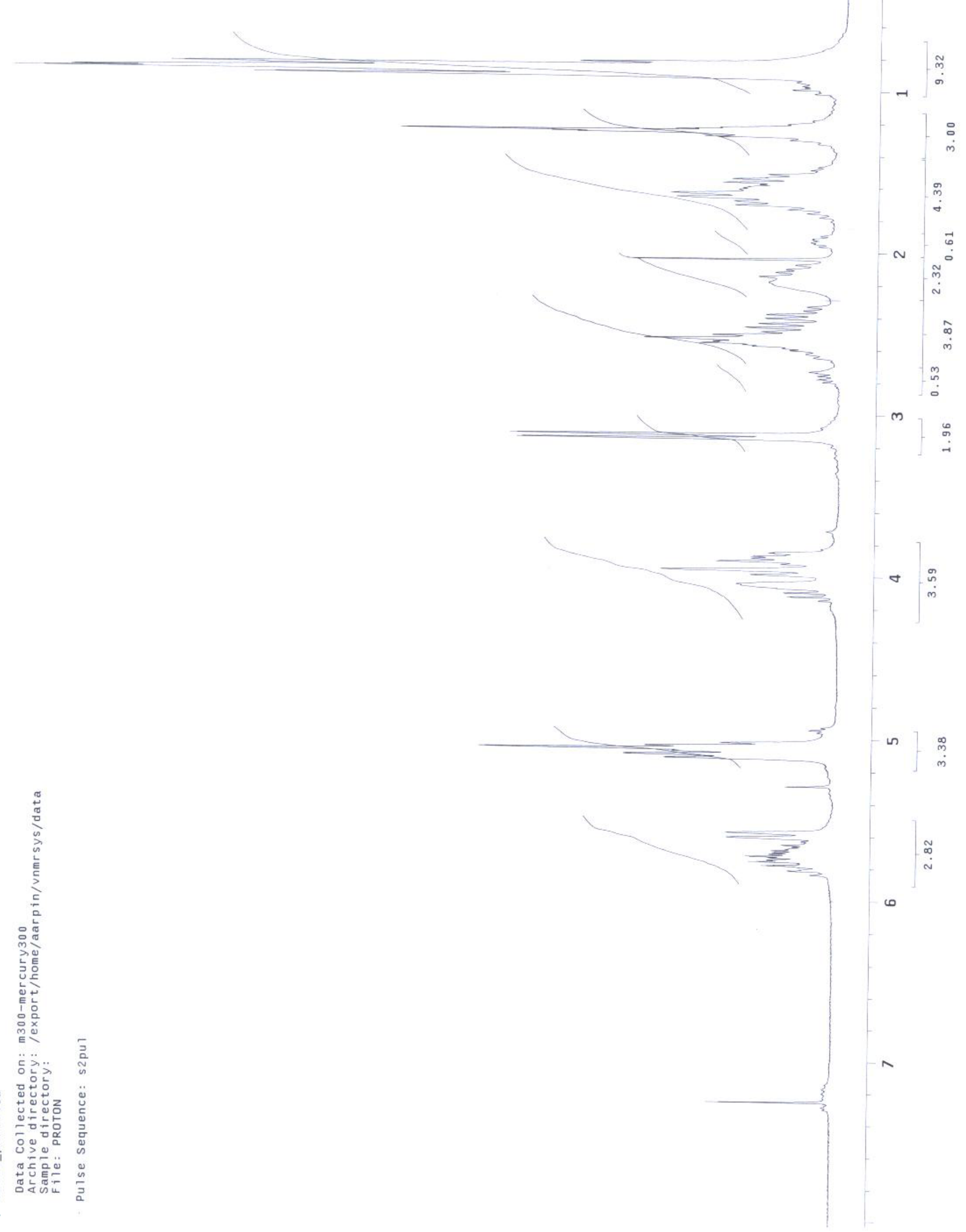


Compound 10a

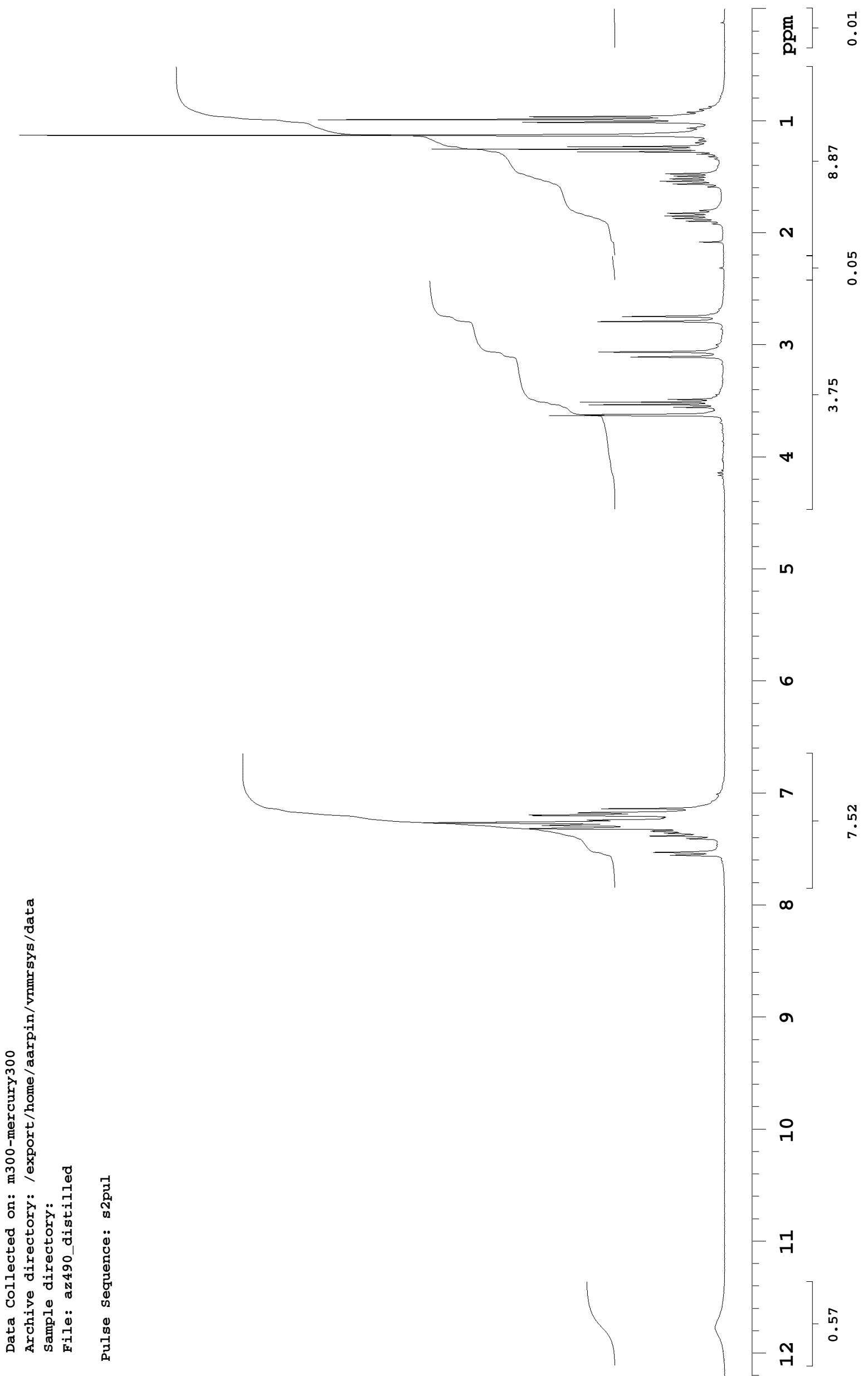


Compound 10b

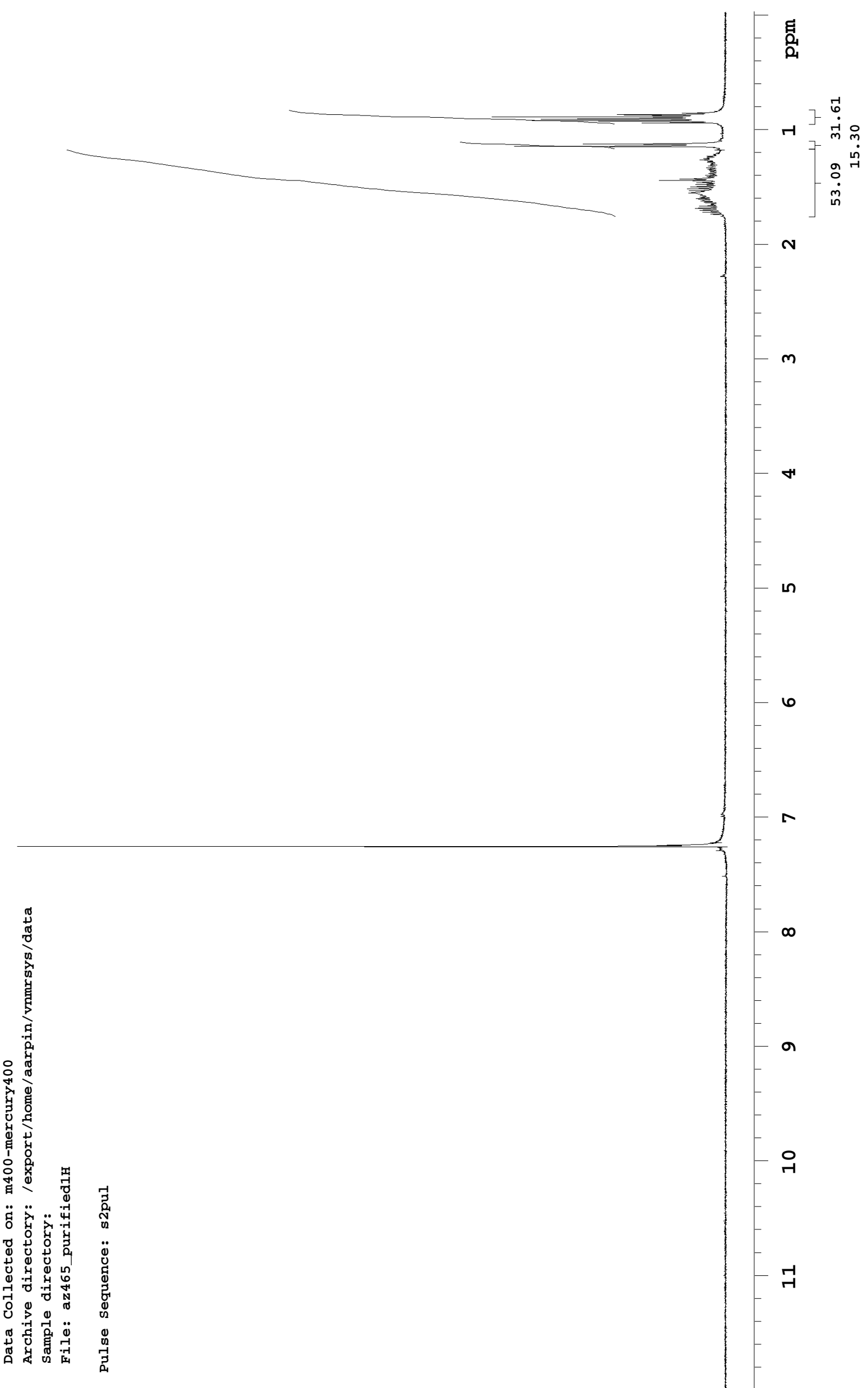


Compound 10j

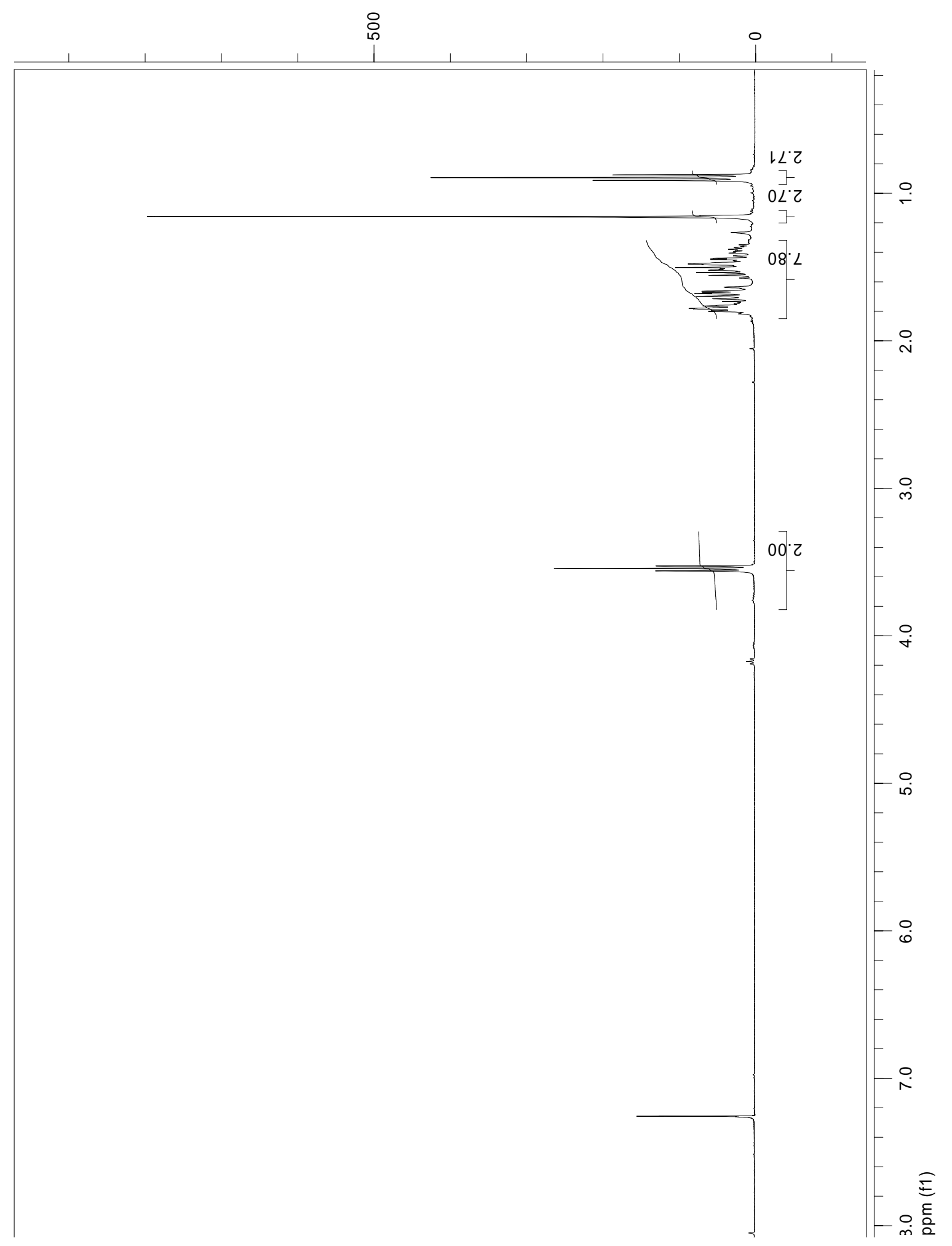


Compound 11a

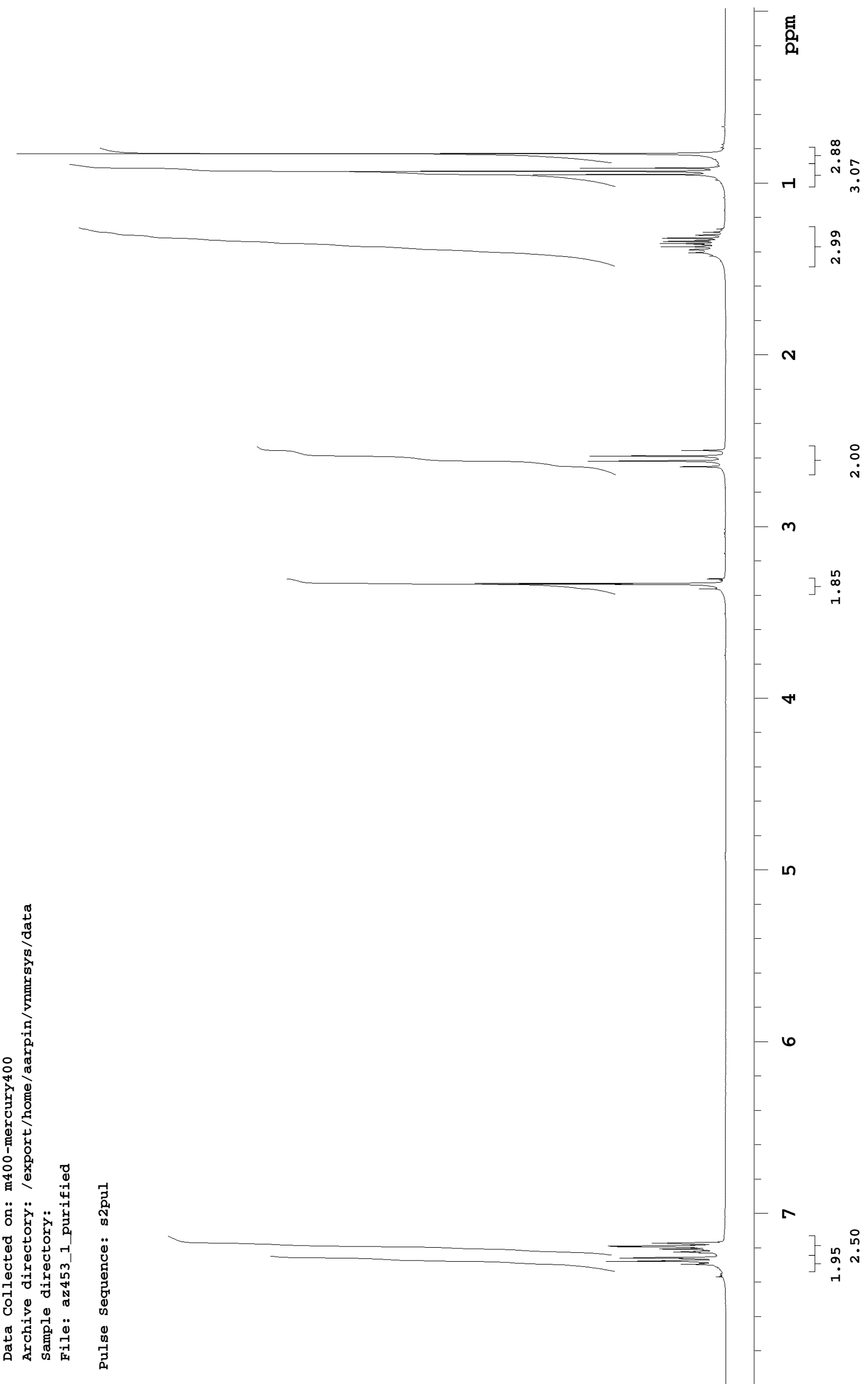


Compound $11 \mathrm{k}$

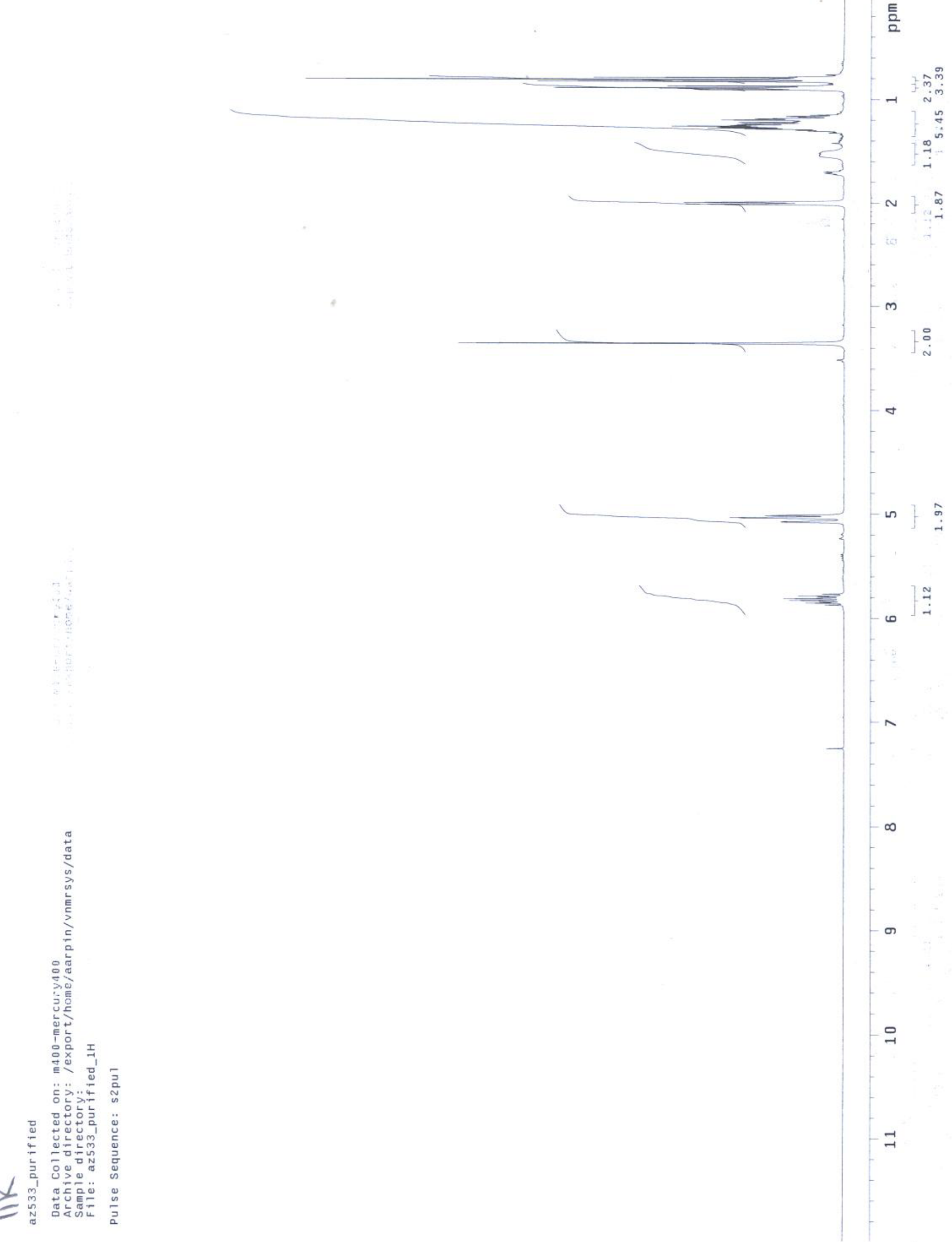

